\title{
Single cell sequencing identifies serine proteases as regulators of myofibroblast differentiation
}

\section{Vera Vorstandlechner}

Medical University of Vienna

Maria Laggner

Medical University of Vienna

\section{Dragan Copic}

Medical University of Vienna

Yiyan Chen

Medical University of Vienna

\section{Bahar Golabi}

Medical University of Vienna

Werner Haslik

Medical University of Vienna

Christine Radtke

Medical University of Vienna

\section{Erwin Tschachler}

Medical University of Vienna

Hendrik Jan Ankersmit

Medical University of Vienna

Michael Mildner ( $\nabla$ michael.mildner@meduniwien.ac.at)

Medical University of Vienna https://orcid.org/0000-0002-6892-925X

\section{Article}

Keywords: skin scarring, serine proteases, myofibroblast differentiation

Posted Date: August 20th, 2020

DOl: https://doi.org/10.21203/rs.3.rs-55007/v1

License: (1) This work is licensed under a Creative Commons Attribution 4.0 International License. Read Full License 
Version of Record: A version of this preprint was published at Nature Communications on October 29th, 2021. See the published version at https://doi.org/10.1038/s41467-021-26495-2. 
1 Single cell sequencing identifies serine proteases as regulators of myofibroblast differentiation 


\section{Abstract}

5 Despite recent advances in understanding skin scarring, mechanisms triggering hypertrophic scar

6 formation are still poorly understood. In the present study we performed single-cell sequencing of

7 mature human hypertrophic scars and developing scars in mice.

8 Compared to normal skin, we found significant differences in gene expression in most cell types

9 present in scar tissue. Fibroblasts (FBs) showed the most prominent alterations in gene expression,

10 displaying a distinct fibrotic signature. By comparing genes upregulated in murine FBs during scar

11 development with genes highly expressed in mature human hypertrophic scars, we identified a group

12 of serine proteases, tentatively involved in scar formation. Two of them, dipeptidyl-peptidase 4

13 (DPP4) and urokinase (PLAU), were further analyzed in functional assays, revealing a role in TGF $\beta 1$ -

14 mediated myofibroblast differentiation and over-production of components of the extracellular matrix

15 (ECM) without interfering with the canonical TGF $\beta 1$-signaling pathway.

16 In this study, we delineate the genetic landscape of hypertrophic scars and present new insights into

17 mechanisms involved in hypertrophic scar formation. Our data suggest the use of serine protease

18 inhibitors for the treatment of skin fibrosis. 
20 Our study deepens the understanding of skin scarring, and provides unique biological insights in

21 tissue fibrosis. We provide data on the transcriptomic landscape of human hypertrophic scars on a

22 single-cell level and investigated scar maturation in vivo. Our study identified numerous genes

23 potentially contributing to scarring in various cell types. Especially a group of serine proteases have

24 been shown to play a key role in scar formation by interfering with non-canonical TGF $\beta$-signaling

25 and prevention of myofibroblast-differentiation. Targeting these proteases with inhibitors might 26 improve scar formation.

\section{Introduction}

30 Hypertrophic scars are a complex medical problem and a significant global disease burden ${ }^{1,2}$. In the

31 western world, an estimated number of 100 million people develop scars every year, approximately

3211 million of which bear keloid scars and 4 million suffer from burn scars ${ }^{1}$. In the USA, an estimated

33 amount of 12 Billion Dollars is spent annually on the treatment of skin scarring ${ }^{3}$. For affected 34 persons, a pathological hypertrophic scar causes significant functional impairment, pain, pruritus, and 35 a reduction in quality of life ${ }^{4,5}$.

36 Wound healing is a tightly coordinated, three-step process, characterized by an acute inflammatory 37 phase, a proliferative phase, and a remodeling phase. Prolonged inflammation results in increased 38 fibroblast (FB) activity, with enhanced secretion of transforming growth factor beta 1 (TGF $\beta 1$ ), TGF $\beta$

392 , insulin-like growth factor (IGF1), and other cytokines ${ }^{6}$. TGF $\beta 1$ drives differentiation of FBs into 40 myofibroblasts, which have a contractile phenotype, are characterized by excessive secretion of

41 ECM-components ${ }^{7}$, and are the major contributors to the formation of hypertrophic scars ${ }^{8}$. Mature 42 hypertrophic scars display strong tissue contraction ${ }^{6}$, and dense, parallel or whorl-like $\mathrm{ECM}^{8}$.

43 Topical silicone application, compression or massage therapy, laser ablation, surgery, and 44 intralesional injection of triamcinolone (TAC), corticosteroids, or 5-Fluorouracil (5-FU) are the most 
commonly used options for prevention or treatment of hypertrophic scars $6,9,10,11$. However, many of

46 these therapies lack evidence of efficacy and safety, and mechanisms of actions are still unclear ${ }^{10,12}$.

47 Recently, several proteases became the focus of drug development in fibrotic diseases, as they have

48 been shown to be involved in ECM-breakdown and the activation of growth factors in tissue

49 remodeling ${ }^{13,14}$. Serine proteases/peptidases constitute a large, diverse group of proteases, divided

50 into 13 clans and 40 families ${ }^{15}$. The group of trypsins comprises proteases contributing to vital

51 processes such as blood coagulation, fibrinolysis, apoptosis and immunity ${ }^{16}$. Members of this family

52 include urokinase, HTRA1/3 (high temperature requirement A1/3 peptidase), several coagulation

53 factors and complement components, PRSS-like serine proteases, granzymes, and cathepsin $\mathrm{G}^{16,17}$.

54 Inhibitors of PLAU have been shown to counteract fibrotic processes in cardiac and pulmonary

55 fibrosis in human in vitro studies and in mouse experiments ${ }^{18,19}$. Recently, the serine protease DPP4

56 became the center of attention, since DPP4 inhibitors (gliptins) have been clinically used for the

57 treatment of diabetes mellitus ${ }^{20}$. DPP4 was also implicated in a variety of fibrotic pathologies,

58 including cardiac, hepatic, renal, and dermal fibrosis $21,22,23,24,25$ and inhibition of DPP4 activity

59 mitigated fibrotic processes in animal models $18,19,26,27,28,29$. However, the contribution to human

60 scar formation and the underlying anti-fibrotic mechanisms are so far not known.

61 Here, we used the new approach of scRNAseq to thoroughly study gene expression and mechanisms

62 involved in hypertrophic scar formation. We aimed to identify new genes regulated in scar tissue, and

63 to uncover potential new targets for drug development towards scar-free wound healing or full

64 reversion of a present scar.

\section{Methods}

\section{Ethical statement}

67 The Vienna Medical University ethics committee approved the use of healthy abdominal skin (Vote

68 Nr. 217/2010) and of scar tissue (Vote Nr. 1533/2017) and all donors provided written informed

69 consent. Animal experiments were approved by the Medical University of Vienna ethics committee

70 and by the Austrian Federal Ministry of Education, Science and Research (Vote Nr. BMBWF- 
$7166.009 / 0075-\mathrm{V} / 3 \mathrm{~b} / 2018)$ and performed in accordance with the Austrian guidelines for the use and

72 care of laboratory animals.

\section{Scar and skin samples}

74 Resected scar tissue was obtained from patients who underwent elective scar resection surgery (donor

75 information is provided in Table S1). Scars were classified as hypertrophic, pathological scars

76 according to POSAS ${ }^{30}$ by a plastic surgeon. Only mature scars, which had not been treated before

77 and persisted for more than two years were used for all experiments. All donors had no known chronic

78 diseases and received no chronic medication. Healthy skin was obtained from female donors between

79 25-45 years from surplus abdominal skin removed during elective abdominoplasty.

\section{Mouse full skin wounding and scar maturation}

81 Female Balb/c mice bred at the animal facility of the Medical University of Vienna (Himberg, Austria)

82 were housed under specific-pathogen-free conditions with $12 \mathrm{~h} / 12 \mathrm{~h}$ light/dark cycles and food and

83 water access ad libidum. For full thickness skin wounds, mice were anesthetized with $100 \mathrm{mg} / \mathrm{kg}$

84 Xylazin and $5 \mathrm{mg} / \mathrm{kg}$ ketamin (both Sigma-Aldrich, St. Louis, MO, USA) intraperitoneally.

85 Postoperative analgesia was provided with $0.1 \mathrm{mg} / \mathrm{kg}$ Buprenorphin (Temgesic $®$, Indivior Inc., North

86 Chesterfield, VA, USA) subcutaneously and $0.125 \mathrm{mg} / \mathrm{ml}$ Piritramid (Janssen-Cilag Pharma, Vienna,

87 Austria) in drinking water ad libidum. A 9x9 $\mathrm{mm}^{2}$ area was marked on shaved backs and excised with

88 sharp scissors. The wounds were left to heal uncovered without any further intervention. Mice were

89 sacrificed 6 or 8 weeks after wounding, and scar tissues were isolated.

\section{Single cell isolation and fluorescence-activated cell sorting (FACS)}

91 Four mm biopsies were taken from human skin, human scar, and from mouse scar tissue, and

92 enzymatically digested with MACS Miltenyi Whole Skin Dissociation Kit (Miltenyi Biotec,

93 Bergisch-Gladbach, Germany) for 2.5h according to the manufacturer's protocol. After processing on

94 a GentleMACS OctoDissociator (Miltenyi), cell suspensions were passed through a $70 \mu \mathrm{m}$ and a 40

$95 \mu \mathrm{m}$ filter and stained with DAPI nuclear dye. Cells were sorted on a MoFlo Astrios high speed cell 
sorting device (Beckman-Coulter, Brea, CA, USA), and only DAPI-negative cells, representing

97 viable cells, were used for single cell RNAseq.

\section{Generation of single-cell gel-bead in emulsions (GEMs) and library preparation}

99 Immediately after sorting, viable cells were loaded on a 10X-chromium instrument (Single cell gene

100 expression 3'v2/3, 10X Genomics, Pleasanton, CA, USA) to generate GEMs. GEM-generation,

101 library preparation, RNA-sequencing, demultiplexing and counting were done by the Biomedical

102 Sequencing Core Facility of the Center for Molecular Medicine (CeMM, Vienna, Austria).

103 Sequencing was performed on an Illumina HiSeq 3000/4000 (Illumina, San Diego, CA, USA) with 3

104 samples per lane, $2 \times 75 \mathrm{bp}$, and paired-end sequencing.

\section{Cell-gene matrix preparation and downstream analysis}

106 Raw sequencing files were demultiplexed, aligned to the human or mouse reference genome

107 (GrCh38/ mm10) and counted using the Cellranger pipelines (Cellranger v3, 10X Genomics). The

108 resulting cell-gene matrices were processed using the 'Seurat'-package (Seurat v3.1.0, Satija Lab,

109 New York, NY, USA) in R-studio in R (R v3.6.2, The R Foundation, Vienna, Austria). From each

110 sample, unwanted variations and low-quality cells were filtered by removing cells with high and low

111 (>3000 and <200) Unique Molecular Identifier (UMI)-counts. First, healthy skin and scar samples

112 were integrated separately to avoid clustering according to donors, and for batch correction.

113 Subsequently, skin and scar data were integrated again into one dataset. Data integration was

114 performed according to the recommended workflow by Butler et al. and Stuart et al. ${ }^{31,32}$. After

115 quality control comparing all donors, we obtained transcriptome data from a total of 25.083 human

116 skin and scar cells, with a median of 24943 reads and 851 detected genes per cell. In mice, we obtained

117 data from 6561 cells 6 weeks after wounding, and 9393 cells 8 weeks after wounding. The samples

118 displayed a median of 24774 reads per cell, and median of 1969 detected genes per cell. After quality

119 control, all mouse samples were integrated together in one integration step. In both datasets,

120 normalized count numbers were used for differential gene expression analysis, for visualization in

121 violin plots, feature plots, dotplots and heatmaps, when displaying features that vary across 
conditions, as recommended by current guidelines ${ }^{33}$. In both datasets, cell types were identified by

123 well-established marker gene expression (Figure S1A, Figure S4A). For identification of

124 differentially expressed genes (DEGs), normalized count numbers were used, including genes present

125 in the integrated dataset to avoid calculation of batch effects. As keratin and collagen genes were

126 previously found to contaminate skin biopsy datasets and potentially provide a false-positive signal

$123^{34}$, these genes (COL1A1, COL1A2, COL3A1 and KRT1 KRT5, KRT10, KRT14, KRTDAP) were

128 excluded from DEG calculation in non-fibroblast clusters (collagens) or non-keratinocyte clusters

129 (keratins), respectively. Moreover, genes Gm42418, Gm17056 and Gm26917 caused technical

130 background noise and batch effect in mouse scRNAseq, as described before ${ }^{35}$, and were thus

131 excluded from the dataset.

\section{Pseudotime analyses}

133 Pseudotime analyses, trajectory-construction and calculation of pseudotime-dependent gene

134 expression were performed in Monocle 2 (Monocle2, v2.14.0, Trapnell Lab, University of 135 Washington, Seatlle, WA, USA) ${ }^{36,37}$. From the integrated FB subset Seurat-object, data were 136 converted into a monocle-compatible CellDataSet. Analysis was then performed according to the 137 recommended pipeline. Cells with mRNA counts two standard deviations above or below the mean 138 were excluded. Size factors and dispersions were estimated, tSNE-reduction and clustering were 139 performed ${ }^{36,37,38}$. As input for pseudotime ordering, differentially expressed genes between skin and 140 scar were used, and trajectories were constructed with DDRTree (R-package 'DDRTree' v0.1.5, by 141 Qi Mao, Li Wang et al., 2015) ${ }^{38}$.

\section{STRING functional protein interaction networks}

143 Protein interaction networks were generated by imputing a list of genes (e.g. upregulated genes in 144 FBs scar vs skin) into the String v11.0 online tool ${ }^{39,40}$ (https://string-db.org/, String consortium, 145 Swiss Institute of Bioinformatics/EMBL Heidelberg/University of Zurich/Novo Nordisk Foundation 146 CPR).

\section{Gene ontology (GO)-networks}


148 Gene lists of significantly regulated genes (adjusted p-value $<0.05$, average log fold change

149 [avg_logFC] $>0.1$ ) were imputed in ClueGO v2.5.5 ${ }^{41}$ plug-in in Cytoscape v3.7.2 ${ }^{42}$ with medium

150 GO-specificity, with GO-term fusion, and only significant $(P$ value $<.05)$ GO-terms are depicted as 151 circles, whereby circle size correlates with $P$ value, and lines represent functional connection of 152 respective GO-terms.

\section{Immunfluorescence stainings}

154 Immmunofluorescence staining on formalin-fixed, paraffin-embedded (FFPE) sections of skin and 155 scar tissue were performed as described previously ${ }^{43}$. Antibodies were used as indicated in Table S2.

156 RNAScope in situ hybridization

157 FFPE-sections of human skin and scar tissue were prepared according to RNAScope (ACDBio, Bio158 Techne, Bristol, UK) pre-treatment protocol, hybridized with probes targeting human DPP4 159 (RNAscope ${ }^{\circledR}$ Probe-Hs-DPP4) and PLAU (RNAscope ${ }^{\circledR}$ Probe-Hs-PLAU), and visualized with 160 RNAscope 2.5 HD Assay - RED as suggested by the manufacturer. Images were acquired by AX70 161 microscope (Olympus, Tokyo, Japan) using the imaging software MetaMorph (Olympus).

\section{Isolation of primary skin fibroblasts}

163 Five mm biopsies were taken from fresh abdominal skin, washed in phosphate-buffered saline (PBS), 164 and incubated in $2.4 \mathrm{U} / \mathrm{ml}$ Dispase II (Roche, Basel, Switzerland) overnight at $4^{\circ} \mathrm{C}$. The next day, 165 epidermis was separated from dermis, and dermis was incubated with Liberase TM (Merck Millipore, 166 Burlington, MA, USA) in Dulbeccos modified eagle medium (DMEM, Thermo Fisher Scientific, 167 Waltham, MA, USA) without supplements at $37^{\circ} \mathrm{C}$ for $2 \mathrm{~h}$. Next, the dermis was passed through 100 $168 \mu \mathrm{m}$ and $40 \mu \mathrm{m}$ filters, rinsed with PBS, and cells were plated in a T175 cell culture flask. Medium 169 was changed the next day, and then every other day until FBs reached $90 \%$ confluency. First passage

170 FBs were used for TGF $\beta 1$-stimulation experiments.

\section{TGF $\beta$ 1-induced myofibroblast differentiation}


172 After the first passage, isolated primary FBs were plated in 6-well plates, supplied with DMEM +

$17310 \%$ fetal bovine serum (FBS, Thermo Fisher Scientific) and 1\% penicillin/streptomycin (Thermo

174 Fisher Scientific) and grown until 100\% confluency. FBs were then stimulated with 10ng/ml TGF $\beta 1$

175 (HEK-293-derived, Peprotech, Rocky Hill, NJ, USA), and with or without DPP4-inhibitor Sitagliptin

$176(10 \mu \mathrm{M})($ Thermo Fisher Scientific) or urokinase-inhibitor BC-11 hydrobromide $(10 \mu \mathrm{M})$ (Tocris by

177 Bio-Techne, Bristol, UK) for 24h. Supernatants were removed and medium and inhibitors were

178 resupplied for another $24 \mathrm{~h}$. Supernatants were collected and stored at $-80^{\circ} \mathrm{C}$ and cells were lysed in

179 1x Laemmli Buffer (Bio-Rad Laboratories, Inc., Hercules, CA, USA) for further analysis.

180 To analyse signaling pathways, FBs were stimulated with TGF $\beta 1$ and inhibitors for $1 \mathrm{~h}$, and then 181 harvested in 1x Laemmli Buffer with protease inhibitor (cOmplete, MiniProtease Inhibitor Cocktail

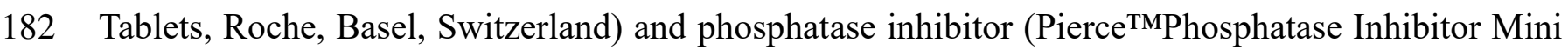
183 Tablets, Thermo Scientific, Waltham, MA, USA).

\section{Western blotting}

185 Primary FBs were lysed in 1x Laemmli Buffer (Bio-Rad Laboratories, Inc.) and loaded on 4-15\% 186 SDS-PAGE gels (Bio-Rad Laboratories, Inc.). Proteins were transferred on a nitrocellulose membrane 187 (Bio-Rad Laboratories, Inc.), membranes were blocked in non-fat milk with 0,1\% Tween 20 for $1 \mathrm{~h}$ 188 (Sigma-Aldrich), and incubated with antibodies as indicated in Table S2 at $4^{\circ} \mathrm{C}$ overnight. After 189 washing, membranes were incubated with horseradish-peroxidase conjugated secondary antibodies 190 as indicated in Table S2 for 1h at room temperature. Signals were developed with SuperSignal West 191 Dura substrate (Thermo Fisher Scientific) and imaged with a Gel Doc XR+ device (Bio-Rad 192 Laboratories, Inc.).

\section{Proteome profiling of signaling pathways}

194 To analyse signaling pathways, we used a proteome profiler for human phospho-kinases (ARY003C, 195 R\&D Systems, Biotechne, Minneapolis, MN, USA) according to the manufacturer`s instructions. 
198 Human pro-collagen Ia1 ELISA (R\&D Systems) and human fibronectin ELISA(R\&D Systems) were 199 performed with supernatants of TGF $\beta$ 1-stimulated FBs according to the manufacturer's manual. 200 Absorbance was detected by FluoStar Optima microplate reader (BMG Labtech, Ortenberg, 201 Germany). 


\section{Results}

\section{The single-cell landscape of hypertrophic scars}

205 To elucidate the complex biological processes of scar formation, we performed droplet-based single-

206 cell transcriptome analysis of human hypertrophic scar tissue and healthy skin ${ }^{44}$ (Figure 1A). In both

207 samples, Unsupervised Uniform Manifold Approximation and Projection (UMAP)-clustering

208 revealed 21 cell clusters, which were further classified as specific cell types by well-established

209 marker genes (Figure S1A), expression patterns of all clusters (Figure S1B), and transcriptional

210 cluster proximity via a phylogenetic cluster tree (Figure 1B). We found seven FB clusters, smooth

211 muscle cells and pericytes (SMC/Peri), three clusters of endothelial cells (EC), and lymphatic

212 endothelial cells (LECs), two clusters of T-cells and of dendritic cells (DC), macrophages (Mac),

213 three keratinocyte (KC) clusters, and melanocytes. All cells of specific subsets were clustered

214 together, and skin and scar samples displayed comparable cellular cluster composition (Figure 1C,

215 D). Only cluster FB1 was mainly present in scar tissue. The clusters of skin and scars showed different

216 relative cell number ratios (Figure 1E, F). Whereas FBs represented $40 \%$ of all cells in healthy skin,

217 a significant increase (53\%) was observed in scar tissue. Similarly, we detected more ECs (16,31\%)

218 in scar tissue as compared to normal skin $(8,1 \%)$. Contrary, the relative numbers of KCs $(5,97 \%)$ and

219 macrophages $(4,42 \%)$ in mature hypertrophic scars were significantly reduced compared to skin $220 \quad(18,97 \%$ and $7,11 \%)$.

221 To identify the strongest differences in gene expression between normal skin and scars, we calculated 222 all up- and downregulated genes for FBs, PCs, ECs, T-cells, DCs and KCs (Figure S2). In general,

223 we identified considerably more up- (Figure 1G) than downregulated genes (Figure 1H), and the most 224 abundant differential gene expression of scar compared to skin (number of differentially expressed 225 genes, $\mathrm{nDEG}$ ) was found in FBs, SMC/pericytes, macrophages, DC1 and $\mathrm{KC1}$ (Figure 1G, H). Genes 226 related to ECM production were mainly overrepresented in FBs, but notably also in PCs and ECs

227 (Figure S2A-C). Several significantly regulated genes with so far undescribed roles in fibrosis and 
scar formation were found in all cell types (Figure S2A-F). These distinctly regulated genes might provide valuable new candidates to understand and modulate skin scarring.

\section{The fibrotic gene expression pattern of fibroblasts in hypertrophic scars.}

232 Since FBs showed strongest gene regulation in our scRNAseq dataset, and have been considered as

233 the major drivers of skin scarring and an important source for myofibroblasts ${ }^{7}$, we focused our further

234 analysis on differences between FBs of healthy skin and hypertrophic scars (Figure 2).

235 After subsetting and reclustering of all FBs, we identified 11 separate clusters (Figure 2A-C) showing

236110 significantly up- and 85 downregulated genes in FBs derived from scar tissue compared to healthy

237 skin. The top 50 differentially up- and downregulated genes are shown in Figure 2D. Interestingly, 238 one FB cluster (FB1) was almost exclusively present in hypertrophic scars, suggesting a specific role 239 in tissue fibrosis. Comparison of FB cluster 1 to all other scar FBs revealed 141 significantly up- and 240179 downregulated genes. The top 50 differentially up- and downregulated genes are shown in Figure

241 2E. Most of the upregulated genes in scar-derived FBs are well-studied in the context of skin scarring 242 and are functionally related to collagens and ECM-modifying genes (Figure S3A, B). However, the 243 interaction on protein level of several genes found here (Figure S3A, B, bold) was hitherto not studied 244 with regard to scar formation.

245 Analysis of the biological processes associated with differentially regulated genes between FB1 and 246 other FB clusters by gene ontology network analysis revealed a strong association of FB1 with TGF $\beta$ 247 signaling (red circles) and ECM-formation (purple circles) (Figure 2F), further corroborating its role 248 in skin fibrosis. In addition, our analysis indicates a role of FB1 in processes important for several 249 other cell types, including platelets, smooth muscle cells and cells of the skeletal system, suggesting 250 paracrine actions of FB1.

251 Pseudotime calculation and trajectory construction effectively identifies possible cell fates and time-

252 regulated genes, even when analyzing cells of only one timepoint ${ }^{36,38}$. Thus, we next sorted human 
skin and scar FBs along a pseudotime axis and constructed trajectories (Figure 3A, B). The

254 trajectories revealed a division at a certain time point where FBs divided into two branches (Figure

$2553 \mathrm{C}$ ). Whereas the majority of FBs preferentially aligned with branch 1 in normal skin (Figure 3D),

256 we observed a significantly longer branch 2 with FBs of hypertrophic scar tissue (Figure 3E). Genes

257 most regulated in a pseudotime-dependent manner in normal skin and hypertrophic scars are shown

258 in Figure $3 \mathrm{~F}$ and $3 \mathrm{G}$. Interestingly, the collagens COL1A1, COL1A2 and COL3A1, known to

259 contribute to all fibrotic processes, are most upregulated at the end of skin pseudotime, but are not

260 among the most pseudotime-regulated genes in scar (Figure 3F,G). In contrast, COL5A1/2, COL8A1,

261 COL11A1 and COL12A1, dominated the late pseudotime-dependent gene expression in scars. The

262 role of these collagens in (hypertrophic) scar is scarcely investigated, and merits further exploration.

263 Together, our trajectory analysis models the temporal dynamics of gene expression in scars and might

264 provide a basis to target respective genes at different stages of scar development.

\section{5 scRNAseq of murine scars identifies genes involved in scar maturation}

266 As our approach so far only gave information on the current state of mature scars, we further

267 investigated mechanisms leading to scar formation and maturation, using a murine full thickness skin

268 wound model. Whereas scar formation and maturation in humans is a long lasting process ${ }^{45}$, it only

269 takes up to 80 days in rodents ${ }^{46}$. Hence, we compared scar formation in mice 6 and 8 weeks after

270 wounding (Figure 4A). Analogously to the human dataset, all cells were clustered, and cell types were

271 identified using established marker genes (Figure S4A), expression patterns of all clusters (Figure

272 S4B) and transcriptome proximity of clusters via a phylogenetic cluster tree (Figure 4B). All clusters

273 aligned homogeneously, and all major skin cell types were represented at both time points (Figure

274 4C, D).

275 In accordance with human scar tissue, 8 weeks old mouse scars contained a higher proportion of 276 murine FBs (mFBs) (32,6\%) compared to 6 weeks old scars $(17,39 \%)$, and more DCs $(9,6$ versus $2776,3 \%)$. In contrast, less pericytes and endothelial cells (2,8 versus 1,5\%) and less keratinocytes $(63,3$ 278 vs 45\%) were present (Figure 4E, F,). We next calculated up- and downregulated genes for FBs, PCs, 
ECs, T-cells, DCs and KCs, comparing 8 weeks to 6-week-old scars (Figure S5A-F). In contrast to

280 human scars, the highest number of differentially expressed genes was found in mFBs and mKCs

281 (Figure 4G,H), which was most likely due to ongoing epidermal tissue regeneration. Surprisingly, the

282 expression of collagens did not change significantly between 6 and 8 weeks in mFBs, and only mECs

283 showed a slightly increased expression of collagens (Figure S5A-C). However, expression of several

284 matricellular proteins and other ECM-components, e.g. Fbln1 (Fibulin1), Ogn (osteoglycin), Lum

285 (Lumican), and Pcolce (Procollagen C-Endopeptidase Enhancer) increased in mFBs during scar

286 maturation (Figure S5A). Together, our scRNAseq identified a gene profile specific for scar 287 maturation in mice.

\section{Serine proteases are strongly upregulated during scar maturation}

290 To identify genes that are crucial for scar maturation, we next compared our human scar data set with genes upregulated in mouse scars 8 weeks after wounding in comparison to mouse scars 6 weeks after wounding (Figure 5A). While in both data sets only one gene (LEPR) was downregulated, 16 genes were mutually upregulated (Figure 5B-D). Stunningly, 5 of these genes (AEBP1, DPP4, HTRA1, PLAU and PRSS23) were members of the superfamily of serine proteases (Figure 5B, C). All five serine proteases were upregulated in scRNAseq in human scar tissue, particularly in FBs, but also in other cell types (Figure 5E-I). AEBP1 and PRSS23 expression also increased in ECs and melanocytes, HTRA1 in ECs and KC3, and PLAU in DCs. Several additional serine proteases, HTRA3 (High-

298 Temperature Requirement A Serine Peptidase 3), DPP7 (Dipeptidyl-Peptidase 7), FAP (Fibroblast 299 activation protein alpha), were upregulated in human scars (Figure S6), and also showed a trend in 300 mouse scars.

301 Analysis of theses serine proteases by pseudotime trajectories in human FBs revealed that their 302 expression mainly increased over time and $A E B P 1$ and HTRA1 significantly enriched at the end of 303 branch 2 (Figure 6A,C). Together, these data suggest a major role of serine proteases in scar formation 304 and/or maturation. 

differentiation and ECM over-production

307 We next wanted to investigate the contribution of the identified serine proteases to scar formation.

308 Since specific inhibitors are commercially available only for DPP4 and urokinase, we focused our

309 further functional studies on these two serine proteases. First, we corroborated our scRNAsec data by

310 analyzing RNA and protein expression of DPP4 and urokinase (PLAU) using in-situ hybridization

311 (Figure 7A, B), and immunofluorescence stainings (Figure 7C, D), respectively. Both methods

312 showed significantly higher expression of DPP4 and PLAU in hypertrophic scars as compared to

313 healthy skin. In addition to dermal FBs, PLAU was also induced in the epidermis of scar tissue. As

314 we have previously identified a strong upregulation of $P L A U$ in scars in DC1 (Figure 5H), the positive

315 cells in the epidermis most likely represent Langerhans cells.

316 As TGF $\beta 1$ is one of the key inducers of scarring and tissue fibrosis, causing differentiation of FBs to

317 pro-fibrotic myofibroblasts $19,47,48,49$, we hypothesized that the serine proteases interact with TGF $\beta$ -

318 signaling. To test this, we stimulated primary FBs from healthy abdominal human skin with active

319 TGF $\beta 1$, which induces expression of alpha-smooth muscle actin ( $\alpha$ SMA), a marker for 320 myofibroblasts. Addition of the DPP4 inhibitor Sitagliptin and the urokinase inhibitor BC11 almost 321 completely abolished the TGF $\beta 1$-mediated myofibroblast-differentiation as shown by $\alpha$ SMA Western

322 blot (Figure 8A, B). Moreover, Sitagliptin and to an even greater extent BC-11 attenuated TGF $\beta 1$ -

323 induced overproduction of the ECM-proteins Collal (Figure 8C, D), and fibronectin (Figure 8E, F)

324 by FBs. These results suggest that serine proteases are involved in the TGF $\beta 1$-induced myofibroblast 325 differentiation. 
327 The serine proteases DPP4 and urokinase do not interfere with canonical TGFß1-signaling

328 pathways

329 To investigate whether the serine protease inhibitors interfere with TGF $\beta 1$ signaling, we investigated

330 the TGF 31 -induced signaling pathways SMAD2 and ERK1/2 ${ }^{50}$. While SMAD2 and ERK1/2

331 phosphorylation was readily induced upon stimulation with TGF $\beta 1$, addition of the inhibitors showed 332 no effects (Figure 9A). In contrast, SMAD1, 5 and 9 showed no activation after TGF $\beta 1$ stimulation

333 (Figure 9A). To further identify other signaling pathways that might be involved in the action of the

334 serine protease inhibitors, we used a signaling proteome profiler, showing that none of the signaling 335 molecules were inhibited by the inhibitors (Figure 9B). Interestingly, the GSK $\alpha / \beta$-pathway, known to 336 attenuate fibrotic processes in the heart ${ }^{51}$ was significantly activated by BC-11 (Figure 9B, Figure 337 S7), suggesting a counter-regulatory action. Together, these data suggest that sitagliptin and BC-11 338 do not interfere with canonical or known non-canonical TGF $\beta 1$ signaling. 
341 Although skin fibrosis has been extensively studied, key mechanisms leading to the development of

342 hypertrophic scars are still not well understood. In addition, treatment options to prevent or treat

343 (hypertrophic) scars are still scarce ${ }^{52}$ and not exceptionally effective. In the present study, we used

344 scRNAseq to elucidate the genetic landscape of hypertrophic scar tissue at a hitherto unmet single

345 cell resolution. We found numerous regulated genes not yet investigated in scar formation and

346 identified serine proteases as regulators of TGF $\beta$-induced myofibroblast differentiation.

347 As expected, our scRNAseq analysis confirmed a plethora of previous studies, but also identified

348 numerous genes, which have so far not been described in the context of skin scarring or tissue fibrosis.

349 For example, the cytokines $M D K$ (midkine) and PTN (pleiotrophin), both involved in cell growth,

350 migration, and angiogenesis ${ }^{53}$, were strongly upregulated in scar FBs. In contrast, SOD2/3

351 (superoxide dismutase 2/3), an enzyme controlling the release of reactive oxygen species (ROS),

352 hence acting as important antioxidant ${ }^{54}$, was strongly downregulated in scar FBs. Interestingly,

353 failure of ROS-scavenging has already been shown to contribute to hypertrophic scar formation ${ }^{55}$.

354 Another interesting and significantly down-regulated gene in scars was $S F N$ (stratifin). As stratifin

355 has been identified as potent collagenase-stimulating factor in FBs, its down-regulation in scars

356 suggests a contribution to the maintenance and/or progression of the fibrotic phenotype by preventing

357 matrix degradation. These and many other novel factors identified in our study could be important,

358 decisive molecules for the development and/or maturation of hypertrophic scars. Thus, our study has

359 built a basis for future studies describing the role of these molecules in skin scarring and tissue

360 fibrosis.

361 Our combined study of human mature hypertrophic scars and scar maturation in mice identified a

362 group of serine proteases as key player for scar development and maturation. Although, DPP4-

363 positive FBs have previously been identified as the main source of ECM production in the skin ${ }^{56}$,

364 and urokinase has been shown to be involved in lung fibrosis ${ }^{19}$, their roles in myofibroblast

365 differentiation and production of ECM are still unclear. Our finding that addition of specific DPP4 
366

367

368

369

370

371

372

373

374

375

376

377

378

and urokinase inhibitors to TGF $\beta 1$-stimulated FBs almost completely inhibited myofibroblast differentiation and up-regulation of matrix proteins was striking. Sitagliptin, the here used DPP4 inhibitor, is an effective drug widely used for the treatment of diabetes mellitus ${ }^{57}$. Based on our study, it might be very interesting to systemically evaluate differences in scar formation and scar quality of diabetic patients treated with either gliptins or other drugs. Furthermore, topical application of gliptins, during the phase of tissue remodeling after full wound closure, might prevent (hypertrophic) skin scarring. As gliptins are already approved for clinical use, an off-label topical application would be a promising step forward to test its efficacy on skin scarring in vivo. Interestingly, the urokinase inhibitor BC-11 showed an even stronger effect on inhibiting TGF $\beta 1$-induced myofibroblast differentiation and matrix production. However, so far it has only been used in vitro, and further in vivo testing for efficacy and safety is still required. Inhibition of urokinase to attenuate tissue fibrosis per se might appear counterintuitive, as urokinase facilitates fibrinolysis and regulates ECM-turnover, eliciting anti-fibrotic action ${ }^{58}$. However, literature on urokinase inhibitors and fibrosis is contradictory. The best investigated endogenous urokinase-inhibitor, plasminogen activator inhibitor1 (PAI-1, SERPINE1), was found to cause excessive matrix deposition after injury ${ }^{59}$. By contrast, and in line with our results, inhibition of urokinase by PAI-1 suppressed profibrotic response in FBs from fibrotic lungs and prevented cardiac fibrosis in mice ${ }^{18}$. Therefore, our study suggests the use of urokinase inhibitor $\mathrm{BC}-11$ as a possible new therapeutic strategy for the treatment of skin scars. Further studies are necessary to fully elucidate its efficacy in vivo.

Strikingly, our analyses revealed no influence of the inhibitors on the canonical TGF $\beta 1$ signaling pathway. As to the anti-fibrotic action of sitagliptin, we have no mechanism to offer yet. Although DPP4 inhibition has previously been demonstrated to directly inhibit canonical TGF $\beta$ signaling via Smad2 in renal fibrosis ${ }^{60}$ and TGF $\beta$-mediated myoFB-differentiation by interfering with ERKsignaling ${ }^{61}$, we were not able to confirm these mechanisms in skin FBs. Possible explanations for alternative the anti-fibrotic action of sitagliptin could be interactions with CXCL12/SDF1 (Stromal derived factor 1), a substrate of DPP4 ${ }^{62}$ which was found to promote scar formation ${ }^{63}$, or with 
392 components of the ECM, such as fibronectin ${ }^{62}$, which have been shown to bind latent TGF ${ }^{19}$, or

393 DPP4 induced cleavage of growth factors or receptors ${ }^{62}$. Deciphering these interactions will be the 394 scope of further mechanistic studies. Regarding BC-11, we found a significant activation of GSK3 $\alpha / \beta$ 395 in TGF $\beta 1$-stimulated FBs. Since GSK3 $\beta$ was previously found to interact with WNT/ $\beta$-catenin 396 signaling ${ }^{64,65}$, and deletion of GSK-3 $\beta$ induced a pro-fibrotic myofibroblast phenotype in isolated 397 cardiac FBs in mice ${ }^{51}$, the activation of GSK3 $\alpha / \beta$ suggests a counter-regulation of TGF $\beta 1$-signaling. 398 It is therefore conceivable that BC-11, at least partially, exerts its anti-fibrotic action via activation of $399 \mathrm{GSK} 3 \alpha / \beta$.

400 Together, our study provides a genetic landscape of hypertrophic scars which is the basis for further 401 investigations on novel genes and fibrotic processes hitherto not studied in the context of skin 402 scarring. In addition, we identified a group of serine proteases involved in scar formation and/or 403 maturation and further in vivo studies will demonstrate the efficacy of serine protease inhibitors on 404 the prevention of scar development and on the improvement of already existing scars. 


\section{Uncategorized References}

410 1. Bayat A, McGrouther DA, Ferguson MWJ. Skin scarring. BMJ : British Medical Journal 326, $411 \quad 88-92$ (2003).

412

413

2. Leavitt T, Hu MS, Marshall CD, Barnes LA, Lorenz HP, Longaker MT. Scarless wound 415

7. Hinz B. Myofibroblasts. Experimental eye research 142, 56-70 (2016).

8. Nabai L, Pourghadiri A, Ghahary A. Hypertrophic Scarring: Current Knowledge of Predisposing Factors, Cellular and Molecular Mechanisms. Journal of Burn Care \& Research 41, 48-56 (2020).

9. Anthonissen M, Daly D, Janssens T, Van den Kerckhove E. The effects of conservative treatments on burn scars: A systematic review. Burns : journal of the International Society for Burn Injuries 42, 508-518 (2016).

10. Kafka M, Collins V, Kamolz LP, Rappl T, Branski LK, Wurzer P. Evidence of invasive and noninvasive treatment modalities for hypertrophic scars: A systematic review. Wound repair and regeneration : official publication of the Wound Healing Society [and] the European Tissue Repair Society 25, 139-144 (2017).

11. Tredget EE, Shupp JW, Schneider JC. Scar Management Following Burn Injury. Journal of burn care \& research : official publication of the American Burn Association 38, 146-147 (2017). 
12. Bao Y, Xu S, Pan Z, Deng J, Li X, Pan F. Comparative Efficacy and Safety of Common Therapies in Keloids and Hypertrophic Scars: A Systematic Review and Meta-analysis. Aesthetic plastic surgery, (2019).

13. Kanno Y. The Role of Fibrinolytic Regulators in Vascular Dysfunction of Systemic Sclerosis.

14. Menou A, Duitman J, Crestani B. The impaired proteases and anti-proteases balance in Idiopathic Pulmonary Fibrosis. Matrix biology : journal of the International Society for Matrix Biology 68-69, 382-403 (2018).

15. Page MJ, Di Cera E. Serine peptidases: classification, structure and function. Cellular and molecular life sciences : CMLS 65, 1220-1236 (2008).

16. Di Cera E. Serine proteases. IUBMB life 61, 510-515 (2009).

17. Rawlings ND, Barrett AJ. MEROPS: the peptidase database. Nucleic Acids Res 27, 325-331 (1999).

18. Gupta KK, Donahue DL. Plasminogen Activator Inhibitor-1 Protects Mice Against Cardiac Fibrosis by Inhibiting Urokinase-type Plasminogen Activator-mediated Plasminogen Activation. 7, 365 (2017).

19. Schuliga M, Jaffar J, Harris T, Knight DA, Westall G, Stewart AG. The fibrogenic actions of lung fibroblast-derived urokinase: a potential drug target in IPF. Scientific reports 7, 41770 (2017).

20. Makrilakis K. The Role of DPP-4 Inhibitors in the Treatment Algorithm of Type 2 Diabetes Mellitus: When to Select, What to Expect. International journal of environmental research and public health 16, (2019).

21. Aroor AR, et al. Dipeptidyl peptidase-4 (DPP-4) inhibition with linagliptin reduces western diet-induced myocardial TRAF3IP2 expression, inflammation and fibrosis in female mice. Cardiovascular diabetology 16, 61 (2017).

22. Hong SK, Choo EH, Ihm SH, Chang K, Seung KB. Dipeptidyl peptidase 4 inhibitor attenuates obesity-induced myocardial fibrosis by inhibiting transforming growth factor-betal and Smad2/3 pathways in high-fat diet-induced obesity rat model. Metabolism: clinical and experimental 76, 42-55 (2017).

23. Kaji K, et al. Dipeptidyl peptidase-4 inhibitor attenuates hepatic fibrosis via suppression of activated hepatic stellate cell in rats. Journal of gastroenterology 49, 481-491 (2014). 
24. Suzuki T, et al. Vildagliptin ameliorates pulmonary fibrosis in lipopolysaccharide-induced lung injury by inhibiting endothelial-to-mesenchymal transition. Respiratory research 18, 177

25. Uchida T, et al. Renoprotective effects of a dipeptidyl peptidase 4 inhibitor in a mouse model

29. $\mathrm{Hu}$ MS, Longaker MT. Dipeptidyl Peptidase-4, Wound Healing, Scarring, and Fibrosis. Plastic and reconstructive surgery 138, 1026-1031 (2016).

30. Fearmonti RM, Bond JE, Erdmann D, Levin LS, Pizzo SV, Levinson H. The modified Patient nonpathologic scarring. Plastic and reconstructive surgery 127, 242-247 (2011).

31. Butler A, Hoffman P, Smibert P, Papalexi E, Satija R. Integrating single-cell transcriptomic data across different conditions, technologies, and species. Nature Biotechnology 36, 411 (2018).

32. Stuart T, et al. Comprehensive integration of single cell data. bioRxiv, 460147 (2018).

33. Luecken MD, Theis FJ. Current best practices in single-cell RNA-seq analysis: a tutorial. Molecular Systems Biology 15, e8746 (2019).

34. Rojahn TB, et al. Single-cell transcriptomics combined with interstitial fluid proteomics defines cell-type-specific immune regulation in atopic dermatitis. Journal of Allergy and Clinical Immunology, (2020). 
532 36. Trapnell $\mathrm{C}$, et al. The dynamics and regulators of cell fate decisions are revealed by pseudotemporal ordering of single cells. Nat Biotechnol 32, 381-386 (2014).

37. Qiu X, Hill A, Packer J, Lin D, Ma YA, Trapnell C. Single-cell mRNA quantification and

38. Qiu X, et al. Reversed graph embedding resolves complex single-cell developmental

40. Snel B, Lehmann G, Bork P, Huynen MA. STRING: a web-server to retrieve and display the repeatedly occurring neighbourhood of a gene. Nucleic Acids Res 28, 3442-3444 (2000).

41. Bindea G, et al. ClueGO: a Cytoscape plug-in to decipher functionally grouped gene ontology and pathway annotation networks. Bioinformatics (Oxford, England) 25, 1091-1093 (2009).

42. Lotia S, Montojo J, Dong Y, Bader GD, Pico AR. Cytoscape app store. Bioinformatics (Oxford, England) 29, 1350-1351 (2013).

43. Gschwandtner $\mathrm{M}$, et al. Histamine suppresses epidermal keratinocyte differentiation and impairs skin barrier function in a human skin model. Allergy 68, 37-47 (2013).

44. Vorstandlechner V, et al. Deciphering the functional heterogeneity of skin fibroblasts using single-cell RNA sequencing. FASEB journal : official publication of the Federation of American Societies for Experimental Biology, (2020).

45. Kant S, van den Kerckhove E, Colla C, van der Hulst R, Piatkowski de Grzymala A. Duration of Scar Maturation: Retrospective Analyses of 361 Hypertrophic Scars Over 5 Years. Advances in skin \& wound care 32, 26-34 (2019).

46. Ferguson MW, O'Kane S. Scar-free healing: from embryonic mechanisms to adult therapeutic intervention. Philosophical transactions of the Royal Society of London Series B, Biological sciences 359, 839-850 (2004).

47. Carthy JM. TGFbeta signaling and the control of myofibroblast differentiation: Implications for chronic inflammatory disorders. Journal of cellular physiology 233, 98-106 (2018).

48. Roberts $\mathrm{AB}$, et al. Transforming growth factor type beta: rapid induction of fibrosis and angiogenesis in vivo and stimulation of collagen formation in vitro. Proceedings of the National Academy of Sciences of the United States of America 83, 4167-4171 (1986). 
49. Lodyga M, Hinz B. TGF-beta1 - A truly transforming growth factor in fibrosis and immunity.

50. Hata A, Chen YG. TGF- $\beta$ Signaling from Receptors to Smads. Cold Spring Harbor

51. Lal H, et al. Cardiac fibroblast glycogen synthase kinase- $3 \beta$ regulates ventricular remodeling and dysfunction in ischemic heart. Circulation 130, 419-430 (2014).

52. Sidgwick GP, McGeorge D, Bayat A. A comprehensive evidence-based review on the role of topicals and dressings in the management of skin scarring. Archives of dermatological research 307, 461-477 (2015).

53. Muramatsu T. Midkine, a heparin-binding cytokine with multiple roles in development, repair and diseases. Proceedings of the Japan Academy Series B, Physical and biological sciences 86, 410-425 (2010).

54. Miao L, St Clair DK. Regulation of superoxide dismutase genes: implications in disease. Free radical biology \& medicine 47, 344-356 (2009).

55. Carney BC, et al. Reactive Oxygen Species Scavenging Potential Contributes to Hypertrophic Scar Formation. The Journal of surgical research 244, 312-323 (2019).

56. Vorstandlechner $\mathrm{V}$, et al. Deciphering the functional heterogeneity of skin fibroblasts using single-cell RNA sequencing. FASEB journal : official publication of the Federation of American Societies for Experimental Biology 34, 3677-3692 (2020).

57. Juillerat-Jeanneret L. Dipeptidyl peptidase IV and its inhibitors: therapeutics for type 2 diabetes and what else? Journal of medicinal chemistry 57, 2197-2212 (2014).

58. Behrendt N. The urokinase receptor (UPAR) and the uPAR-associated protein (uPARAP/Endo180): membrane proteins engaged in matrix turnover during tissue remodeling. Biological chemistry 385, 103-136 (2004).

59. Rabieian R, Boshtam M, Zareei M, Kouhpayeh S, Masoudifar A, Mirzaei H. Plasminogen Activator Inhibitor Type-1 as a Regulator of Fibrosis. 119, 17-27 (2018). 
616 61. Thielitz A, et al. Inhibitors of dipeptidyl peptidase IV-like activity mediate antifibrotic effects in normal and keloid-derived skin fibroblasts. The Journal of investigative dermatology $\mathbf{1 2 8}$, 855-866 (2008).

62. Elmansi AM, et al. What doesn't kill you makes you stranger: Dipeptidyl peptidase-4 (CD26) proteolysis differentially modulates the activity of many peptide hormones and cytokines generating novel cryptic bioactive ligands. Pharmacology \& therapeutics 198, 90-108 (2019).

64. Guo Y, et al. Entanglement of GSK-3 $\beta$, $\beta$-catenin and TGF- $\beta 1$ signaling network to regulate myocardial fibrosis. Journal of molecular and cellular cardiology 110, 109-120 (2017).

65. Vallée A, Lecarpentier Y, Guillevin R, Vallée JN. Interactions between TGF- $\beta 1$, canonical 
638 The authors declare no conflict of interest.

639

\section{Acknowledgements and funding}

641 This research project was financed in part by the FFG Grant "APOSEC” (852748 and 862068; 2015 642 2019), by the Vienna Business Agency “APOSEC to clinic," (ID 2343727, 2018-2020), and by the

643 Aposcience AG under group leader HJA. MM was funded by the Sparkling Science Program of the 644 Austrian Federal Ministry of Education, Science and Research (SPA06/055). We thank HPH for his 645 belief in this private-public partnership to augment patients' health. We thank the Biomedical 646 Sequencing Facility, Center for Molecular Medicine, Vienna, Austria, for the processing and 647 sequencing of our samples. The authors acknowledge the core facilities of the Medical University of 648 Vienna, a member of Vienna Life Science Instruments.

\section{Author contributions}

651 MM, HJA, ET and VV provided study conception and design; WH and CR provided patient sample 652 material; HJA and MM acquired funding; VV, DC, YC and BG conducted experiments and prepared 653 samples; VV performed data analysis, visualization and figure design; VV, ML, ET, and MM 654 participated in data interpretation; VV, ML and MM drafted the manuscript. All authors reviewed the 655 manuscript. 
Figure 1

(A)

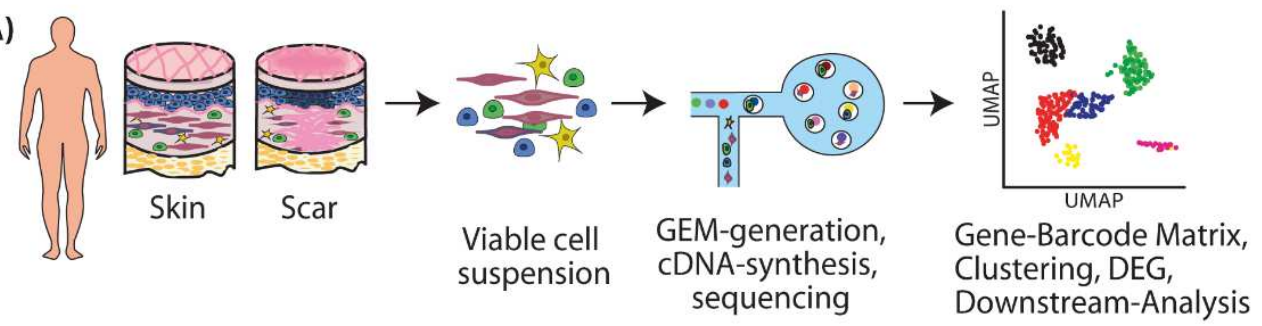

(B)

(D)

Clustering, DEG,

Downstream-Analysis
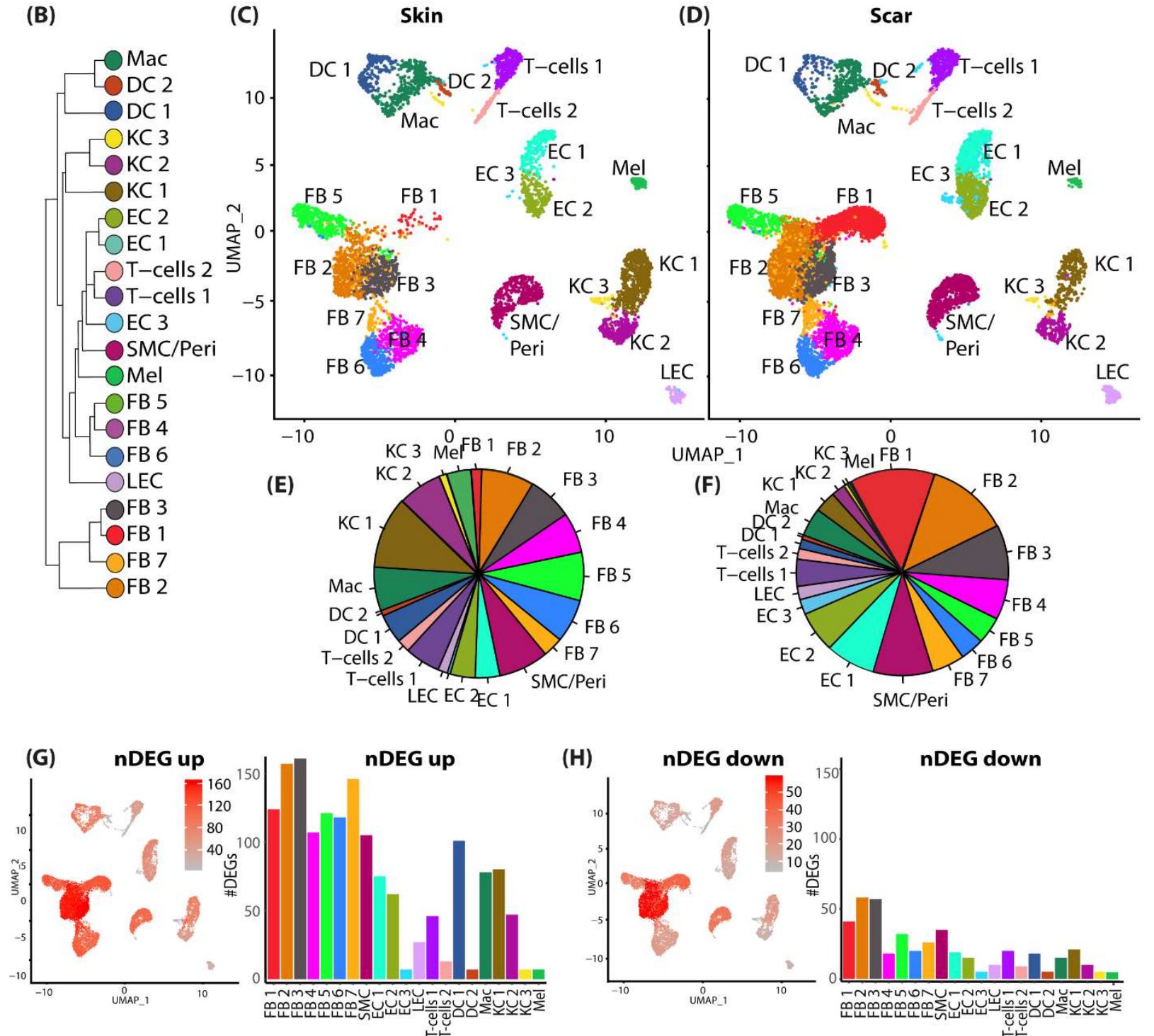

659 Figure 1: Characterization of human skin and scar samples with scRNAseq identifies specific cell clusters and a distinct fibrotic gene signature.

661 A) Illustration of workflow of scRNAseq in human skin and scar samples. B) Phylogenetic clustertree 662 calculated unsupervised based on UMAP-clustering. C, D) UMAP-plots of human skin and scar 663 samples, split by tissue, after integration of all samples, identifying seven fibroblast clusters (FB1-7), 664 smooth muscle cells and pericytes (SMC/Peri), endothelial cells $(\mathrm{EC} 1+2)$, lymphatic endothelial cells 665 (LEC), T-cells, macrophages (Mac), dendritic cells (DC1+2), three keratinocyte clusters (KC1-3), 666 and melanocytes (Mel). E, F) Pie charts showing ratios of cell clusters in skin and scars. Feature plots 667 and bar graphs of number of differentially expressed genes (nDEG) per cluster of G) up- and $\mathrm{H}$ ) 668 downregulated genes. DEGs were calculated per cluster comparing scar versus skin using Wilcoxon 
669 rank sum test, including genes with average logarithmic fold change (avglogFC) of $>0.1$ or $<-0.1$ 670 and Bonferroni-adjusted p-value $<0.05$. Feature plots show projection of nDEG onto the UMAP-plot, 671 color intensity represents nDEG. Bar graphs show absolute numbers of nDEG per cluster, y-axis 672 represents nDEG. UMAP, uniform manifold approximation and projection.

673 
(A)

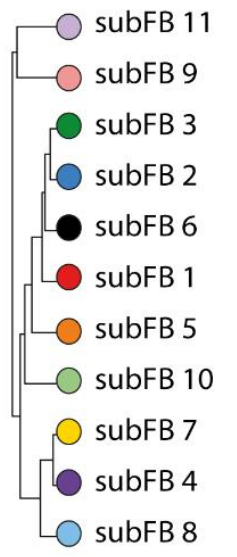

(B)

Skin

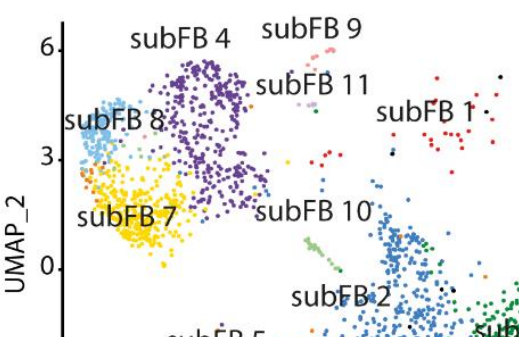

(C)

Scar

Figure 2
(D) Skin FBs Scar FBs
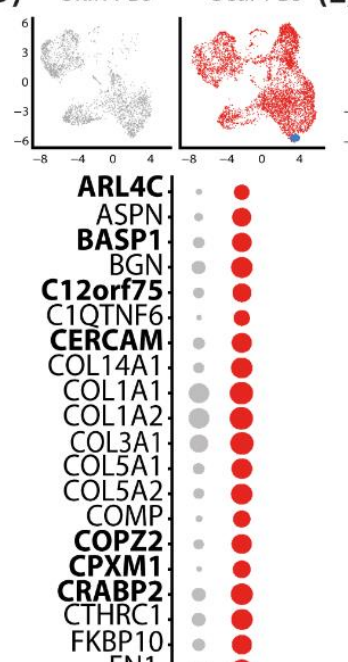$$
\text { HSPA }
$$$$
\text { FT }
$$$$
\text { HSPATN }
$$$$
\text { HSPA1B. }
$$$$
\text { HSPA }
$$$$
\text { KDELR }
$$$$
\text { KLF2. - }
$$$$
\text { KR }
$$$$
\text { KRT14. }
$$$$
\text { LOX. }
$$$$
\text { LUM }
$$$$
\text { MAFB }
$$$$
\text { MMP23 }
$$$$
\text { MXRA5 }
$$

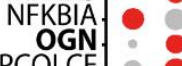$$
\text { PPIC. }
$$$$
\text { PRSS23. }
$$$$
\text { PTN } 0
$$$$
\text { RCN3 }
$$$$
\text { RPLP1 }
$$$$
\text { SFRP2 }
$$$$
\text { SMOC2. }
$$$$
\text { SPARC }
$$$$
\text { THY1 }
$$$$
\begin{array}{r}
\text { TPM2 } \\
\text { SPAN13 }
\end{array}
$$

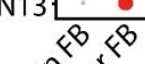$$
s+\sigma_{5} c^{\alpha}
$$

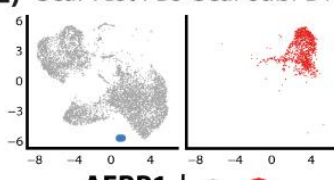

(F)

COPZ

CST3

CXCL14 19 plasminogen

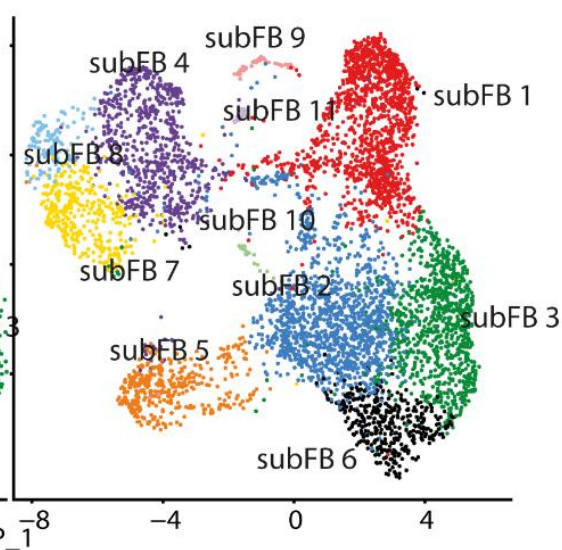

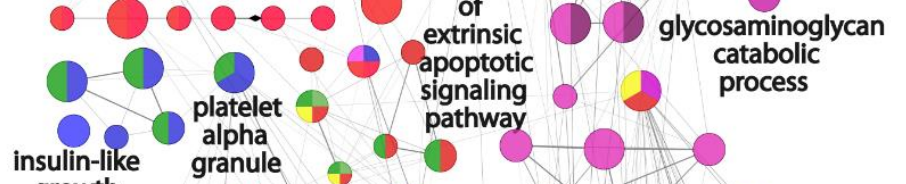

growth

factor

binding

protein

complex platelet
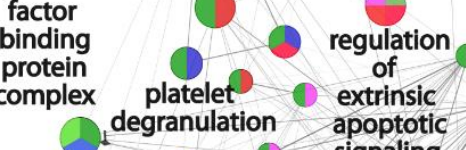

plasminogen

signaling
pathway

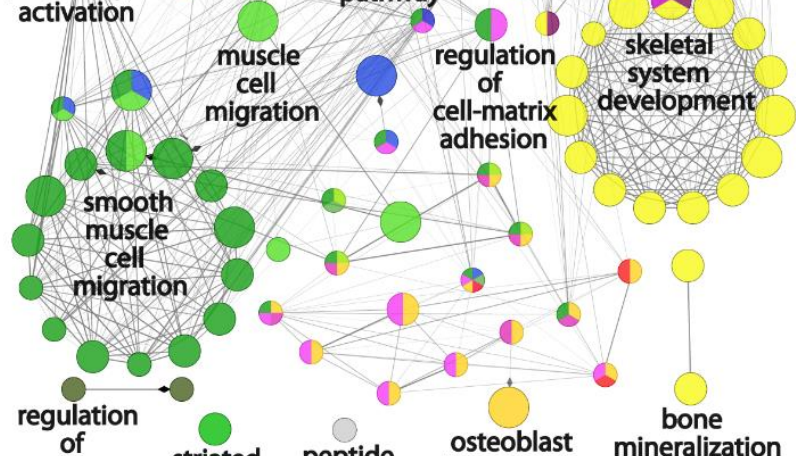

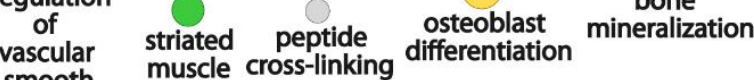

smooth cell

cell development

proliferation

675 Figure 2: Subset analysis of fibroblasts 
676 A) Phylogenetic clustertree calculated unsupervised based on UMAP-clustering of subsetted 677 fibroblasts only. B, C) UMAP-plots of re-clustered skin and scar fibroblasts, split by tissue, re678 clustering identified 11 fibroblast clusters (subFB1-11). D) Feature plots illustrating computational 679 basis for dotplots, dotplots of top 50 regulated genes (according to lowest adjusted p-value) 680 comparing scar FBs versus skin FBs. E) Dotplot of top 50 regulated genes (according to lowest 681 adjusted p-value) cluster subFB1 compared to all other scar FBs. F) Gene ontology-term network was 682 calculated based on significantly upregulated (adj. p-val $<0.05$, avg.logFC $>0.1$ ) genes comparing 683 subFB1 to all other scar FBs. Gene list was imputed in ClueGO plug-in in Cytoscape with medium 684 GO-specificity, with GO-term fusion, only significant $(P$ value $<.05)$ GO terms are shown. Circle size 685 correlates with $P$ value, lines ("edges") represent functional connection of respective GO terms. Red 686 circles represent association of GO-term with TGF $\beta$-signaling, purple, with extracellular matrix, 687 green, with smooth muscle differentiation, blue, with signaling factors, and yellow with bone 688 formation and -development. UMAP, uniform manifold approximation and projection. 


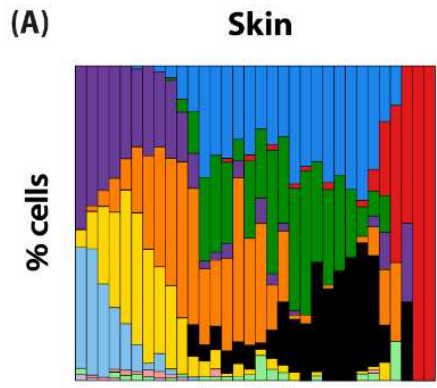

Pseudotime
(B)

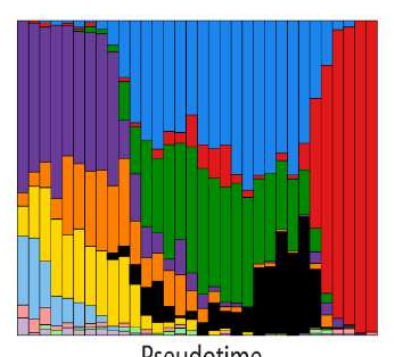

Pseudotime

(C)

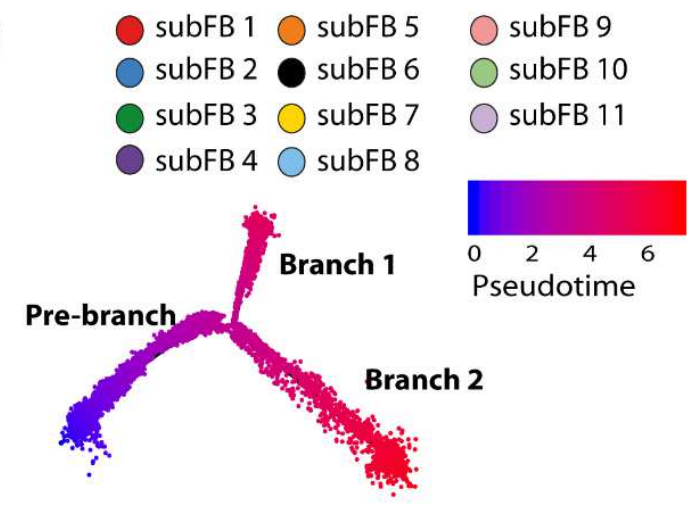

(D)

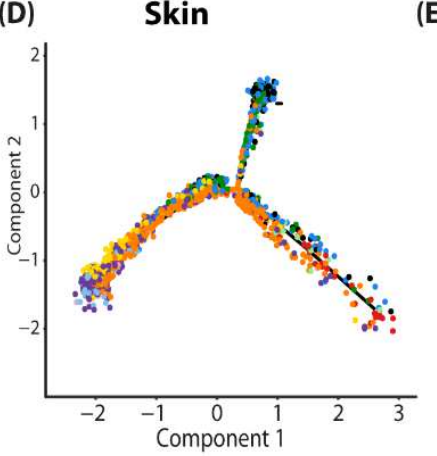

(E)
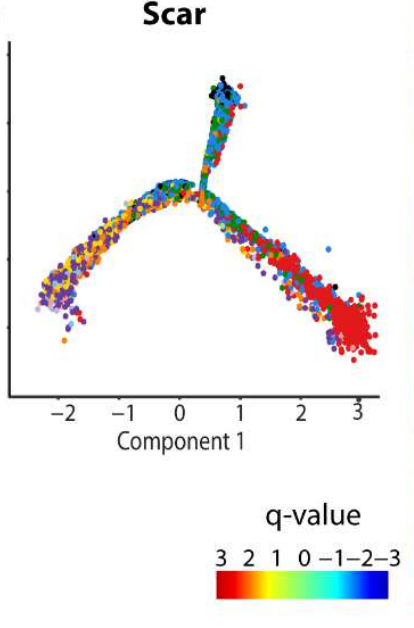

(F)

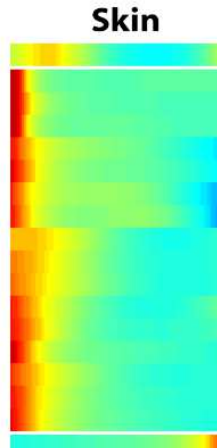

(G)

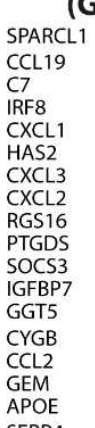

SFRP4

COL3A1

COLSA1

COL1A2

CLEC3B

IGFBPG

ELN

CIOTNF3

CERCAM

SH3BGRL3

SH3BG

PRG4
CD34

TNXB

CD55

CPE
MFAP5
CILP

HAS1

SPARC

ECM1
CTSK

CTSK

SEMA3B

SLC29A1

SLC29A1

MATN

TSPAN8

CFD

CD70

PCSK1N
FAM180B

FAM180B

COL12A1

WISP2

SLPI

LOX

MMP2

MMP2

CTHRC1

FBLN2

TIMP2

QPCT

DCN
AEBP1
PDGFRL

PDGFRL

DPP4

FBLN1
SCARA5

SCARA5
MFGE8
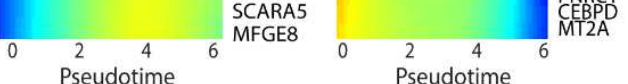

690

691 Figure 3: Pseudotime analysis of human scar FBs identifies cell fates and time-regulated genes

692 A, B) Ordering skin and scar FBs along a pseudotime axis. X-axis, pseudotime. y axis, \% of cells in 693 respective pseudotime-bin. Cell trajectory with pre-branch and branches is shown. Color code 694 represents pseudotime progression. C, D) Cell trajectories were calculated based on pseudotime 695 values, split by tissue. E, F) Heatmaps of pseudotime-dependent gene expression in skin and scar. 696 Colors represent q-value, the expression of the respective gene in relation to pseudotime. 
(A)

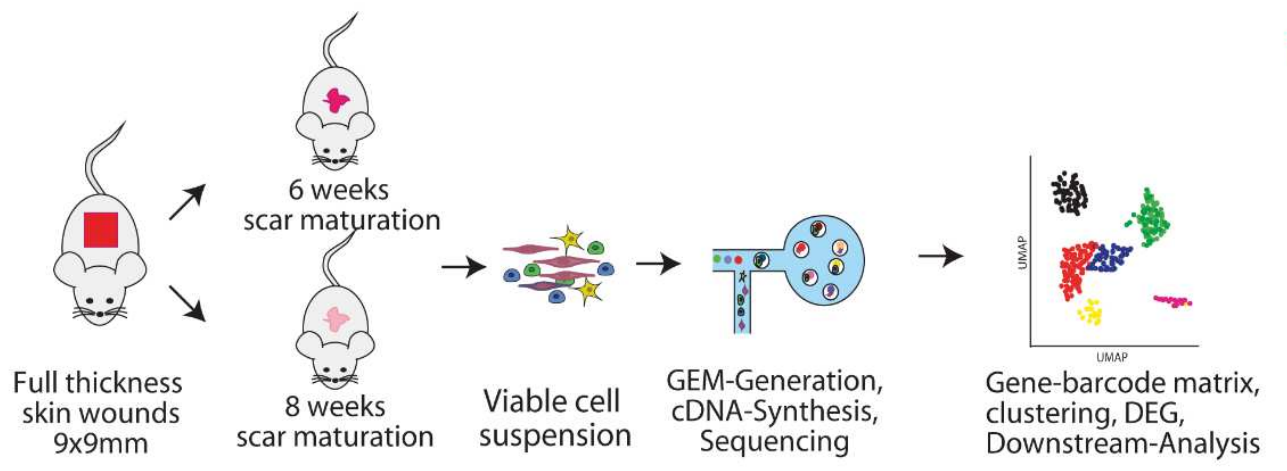

(B)

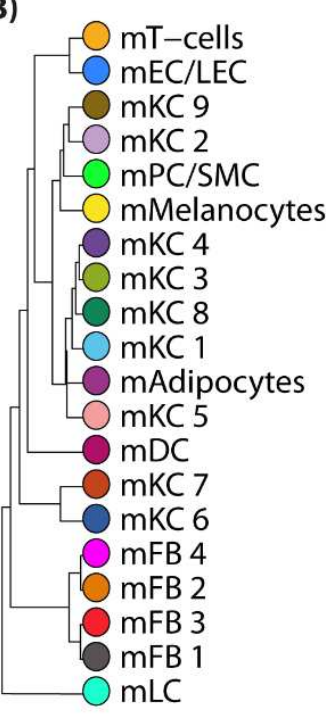

(C) 6 weeks

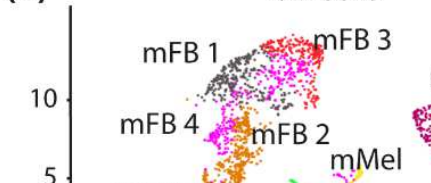

$v_{1}^{5} \mathrm{mEC/LEC} \mathrm{mPC} / \mathrm{SMC}^{\mathrm{mMC}} \mathrm{mLC}$ mT-cells

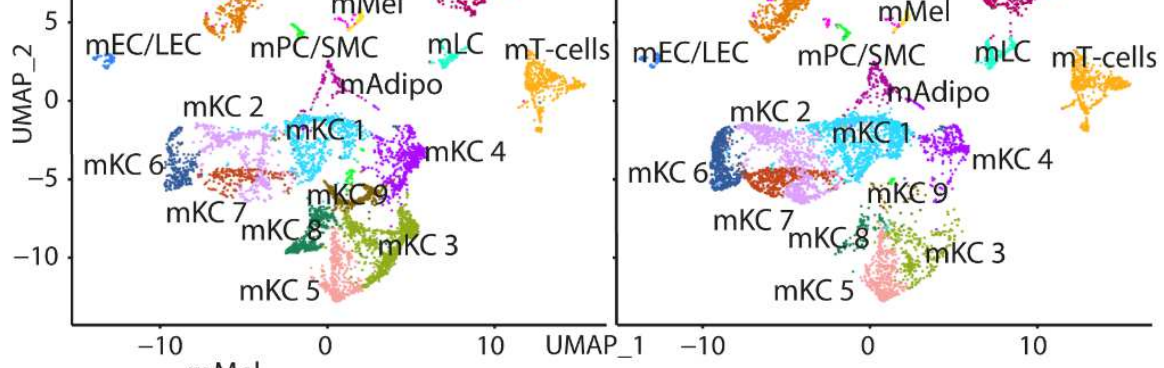

(D)

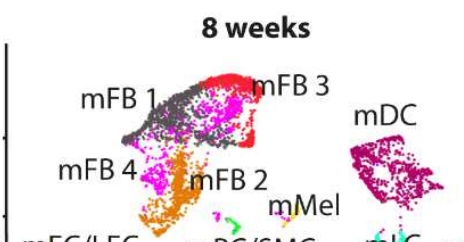

(E)

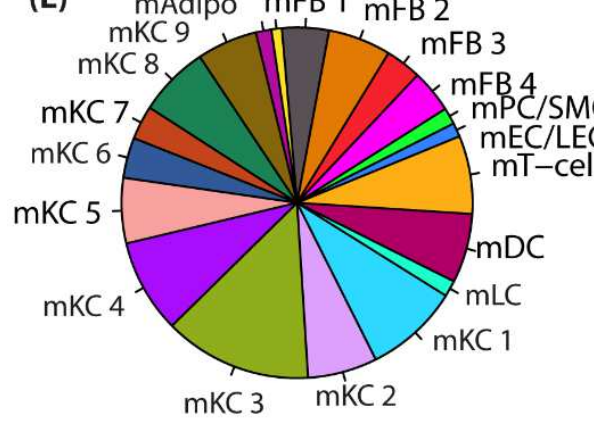

(F) $\quad$ mAdipo $\mathrm{mFB} 1$

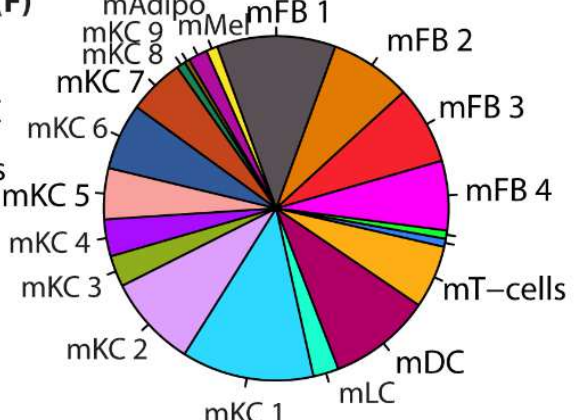

(G)
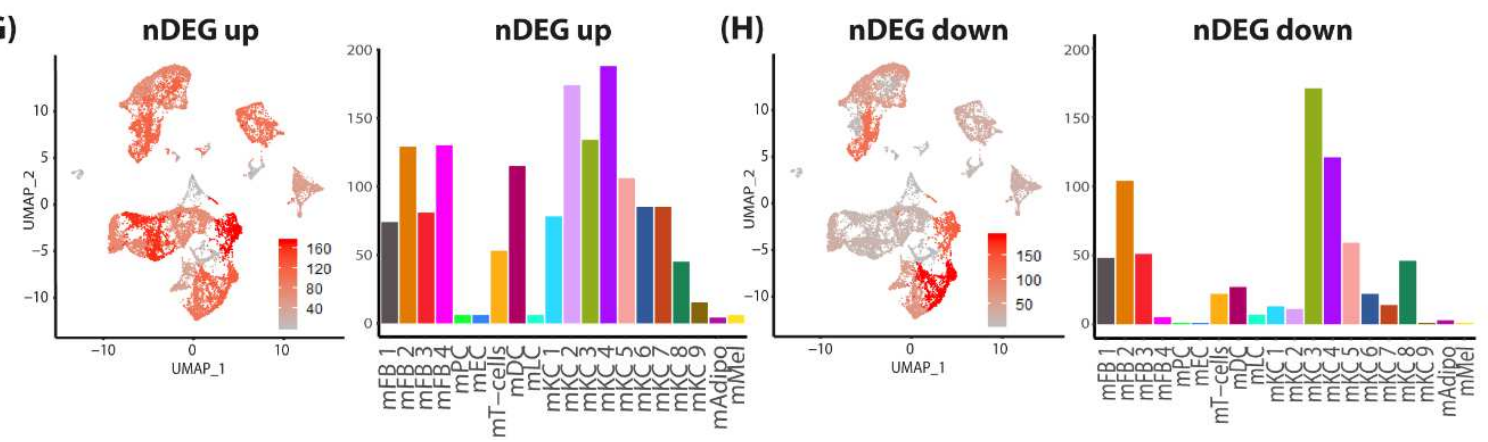

700 Figure 4: Two-timepoint mouse scar model identifies genes regulated in scar maturation

A) Illustration of workflow of mouse scar model and two-timepoint scRNAseq. Two mice were analyzed per timepoint. B) Phylogenetic clustertree calculated unsupervised based on unsupervised UMAP-clustering. C, D) UMAP-plots of mouse scar tissue, split by timepoint, after integration of all samples, identifying four fibroblast clusters (mFB1-4), smooth muscle cells and pericytes (mPC/SMC), endothelial cells and lymphatic endothelial cells (mEC/LEC), T-cells, dendritic cells $(\mathrm{mDC})$, Langerhans cells (mLC), nine keratinocyte clusters (KC1-9), adipocytes (mAdipo) and melanocytes (Mel). E, F) Pie charts show relative numbers of cells in clusters, split by timepoint. up- and H) downregulated genes per cluster. DEGs were calculated per cluster comparing 8 weeks vs 
7106 weeks old scars using Wilcoxon rank sum test, including genes with average logarithmic fold 711 change (avg_logFC) of $>0.1$ or $<-0.1$ and Bonferroni-adjusted p-value $<0.05$. Feature plots show 712 projection of $\mathrm{nDEG}$ onto the UMAP-plot, color intensity represents $\mathrm{nDEG}$. Bar graphs show absolute 713 number of nDEG per cluster, y-axis represents nDEG. UMAP, uniform manifold approximation and 714 projection.

715 
(A)
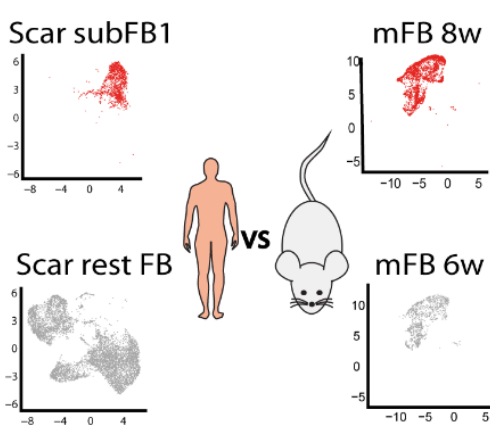

(B)

subFB1 vs rest scarFB $\quad \mathrm{mFB} 8 \mathrm{w}$ vs $\mathrm{mFB} 6 \mathrm{w}$

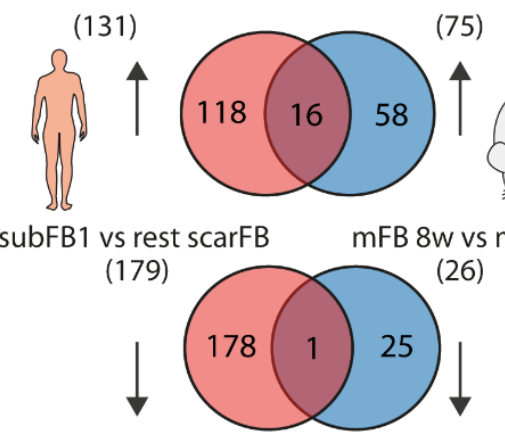

(C)

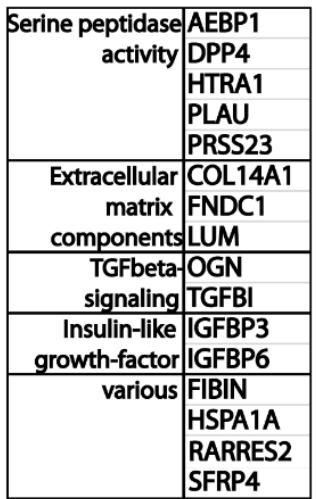

(D)

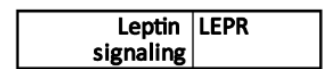

(E)

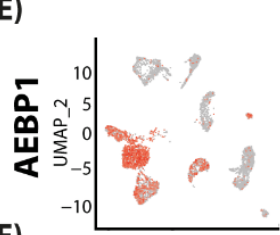

(F)

Skin

(G)
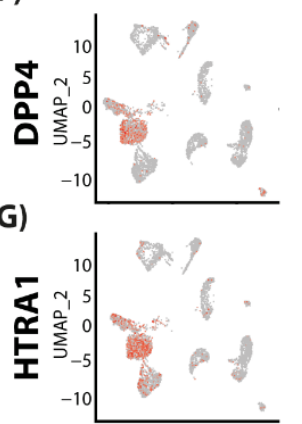

(H)

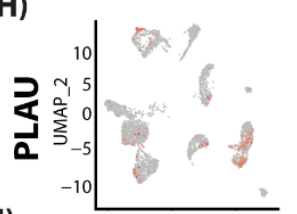

(I)

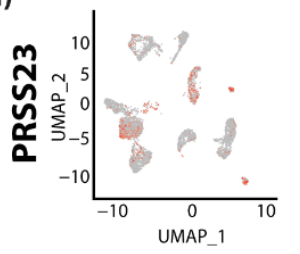

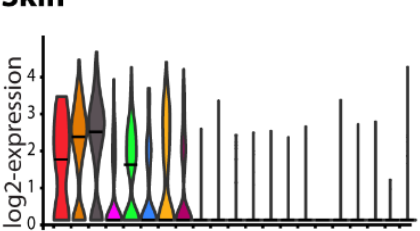
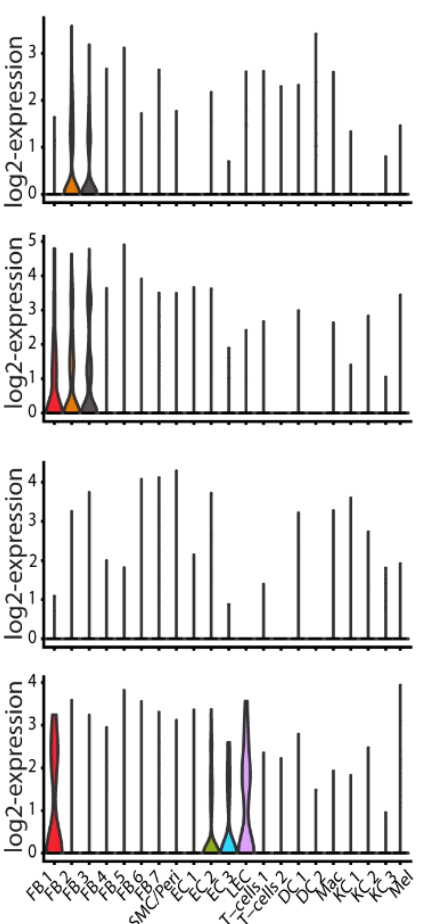

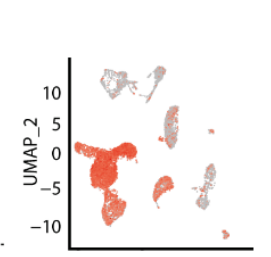

Scar
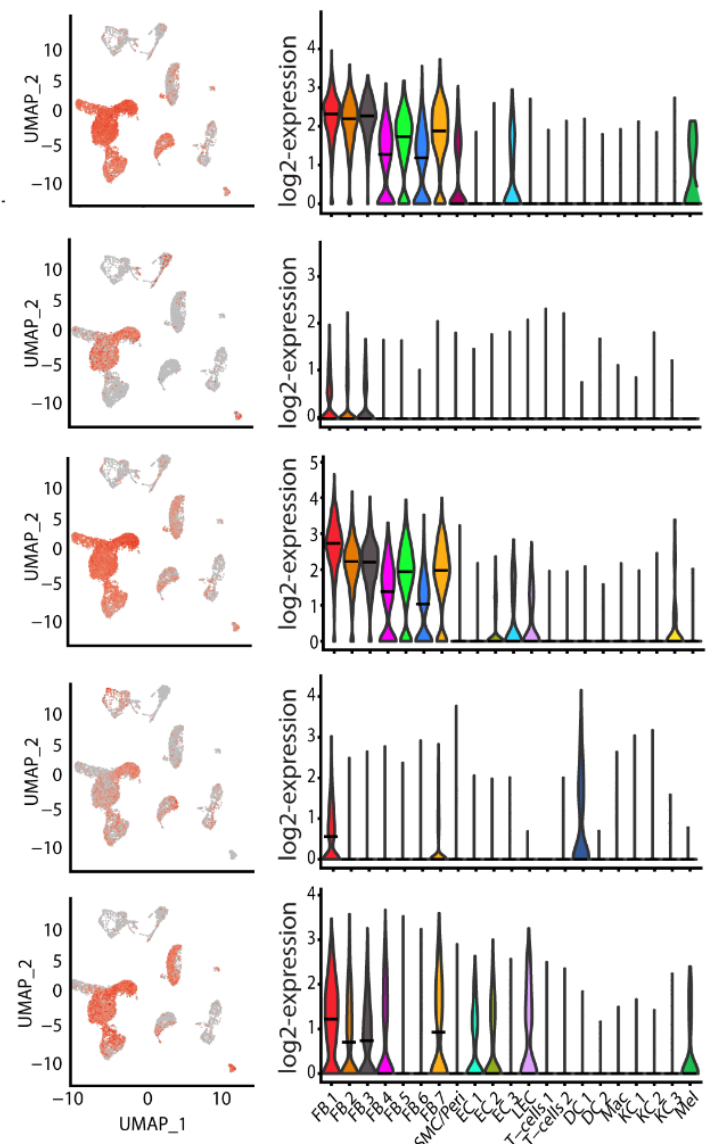

717 Figure 5: Comparing human scar gene expression and mouse scar maturation identifies mutual 718 drivers of skin fibrosis

719 A) Illustration of computational basis for comparison human and mouse. Human cluster subFB1 vs 720 rest scar FBs significantly (adj. p-value $<0,05$ ) regulated genes were compared with mouse scar FBs

7218 weeks vs 6 weeks significantly regulated genes. B) Venn diagrams of human and mouse up- (upper 722 panel) and down- (lower panel) regulated genes. C) Table of mouse and human mutually up and D) 723 downregulated genes. E-I) Feature plots and violin plots of serine proteases in human skin and scar. 724 AEBP1 (adipocyte enhancer binding protein 1), DPP4 (dipeptidyl-peptidase 4), HTRA1 (High725 Temperature Requirement A Serine Peptidase 1), PLAU (urokinase), PRSS23 (Serine protease 23). In 726 violin plots, dots represent individual cells, $y$-axis represents $\log 2$ fold change of the normalized genes 727 and log-transformed single-cell expression. Vertical lines in violin plots represent maximum 
728 expression, shape of each violin represents all results, and width of each violin represents frequency 729 of cells at the respective expression level. In feature plots, normalized log expression of the respective 730 gene is mapped onto the UMAP-plot. Color intensity indicates level of gene expressions. UMAP, 731 uniform manifold approximation and projection.

732 
Figure 6
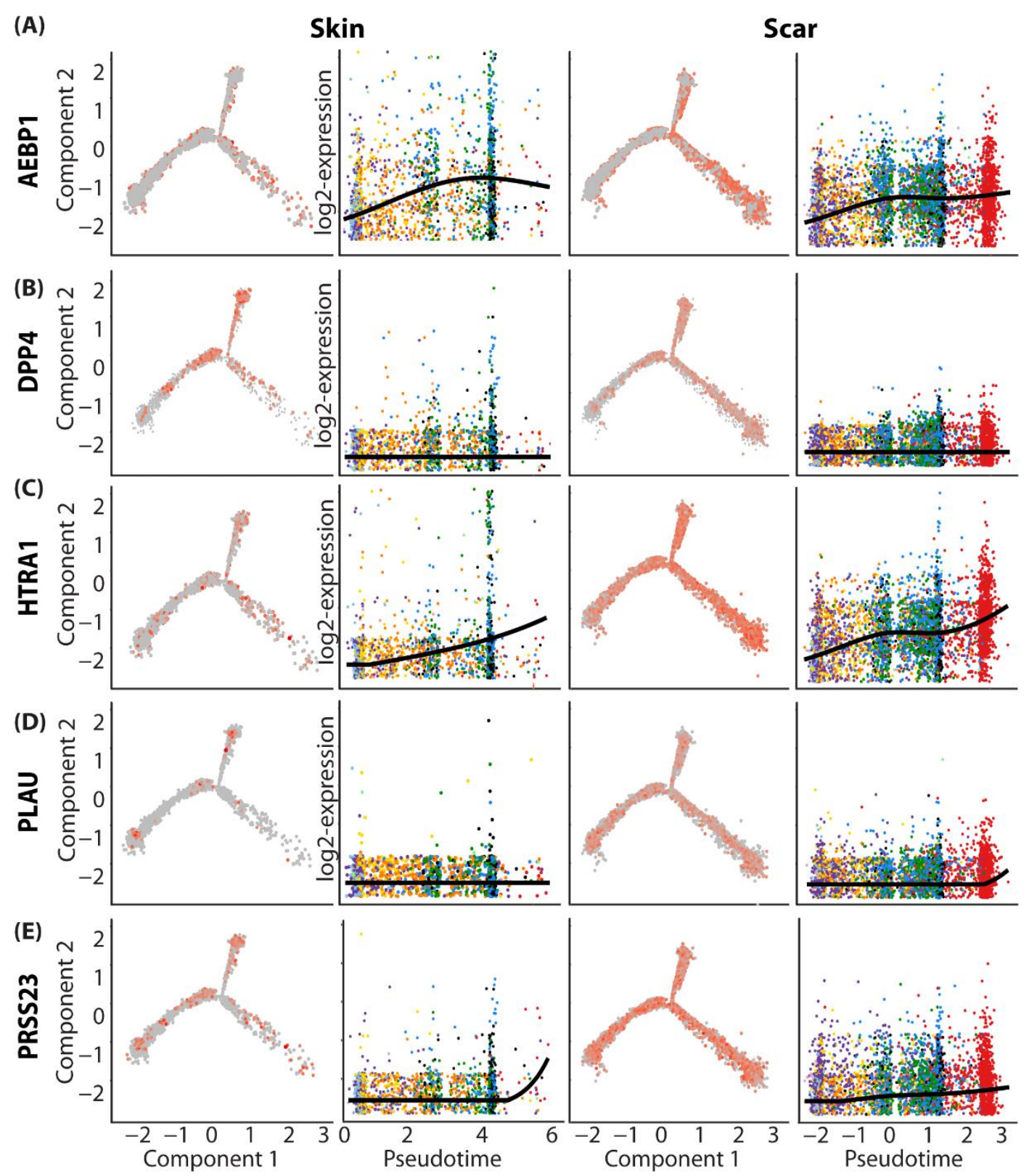

734 Figure 6: Pseudotime analysis corroborates the putative role of serine proteases as drivers of 735 scar maturation

736 A-E) Trajectory plots and pseudotime plots of Serine proteases in skin and scar FBs. AEBP1 737 (adipocyte enhancer binding protein 1), DPP4 (dipeptidyl-peptidase 4), HTRA1 (High-Temperature 738 Requirement A Serine Peptidase 1), PLAU (urokinase), PRSS23 (Serine protease 23). In trajectory 739 plots, normalized log expressions are plotted on the trajectories, split by skin and scar. In pseudotime 740 plots, normalized log expressions are plotted against the pseudotime axis, and a spline curve 741 represents expression dynamics over pseudotime. Y-axis, normalized log expression of respective 742 gene, $x$-axis, pseudotime. 
Skin

(A)

(B)

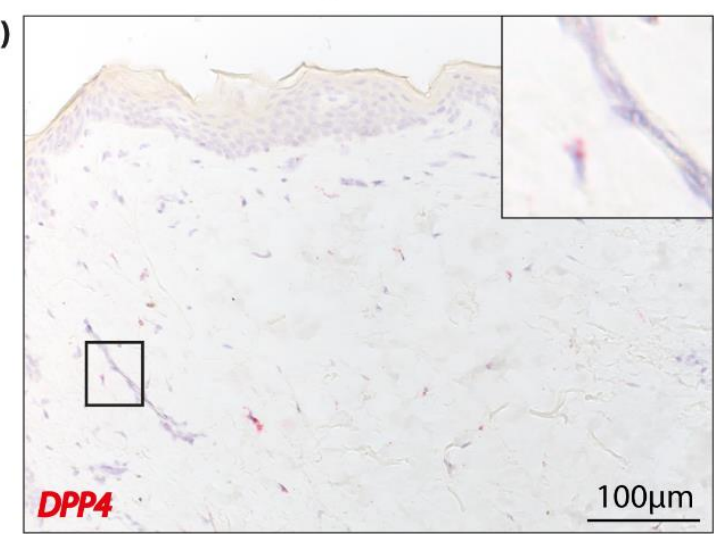

\section{PLAU}

(C)

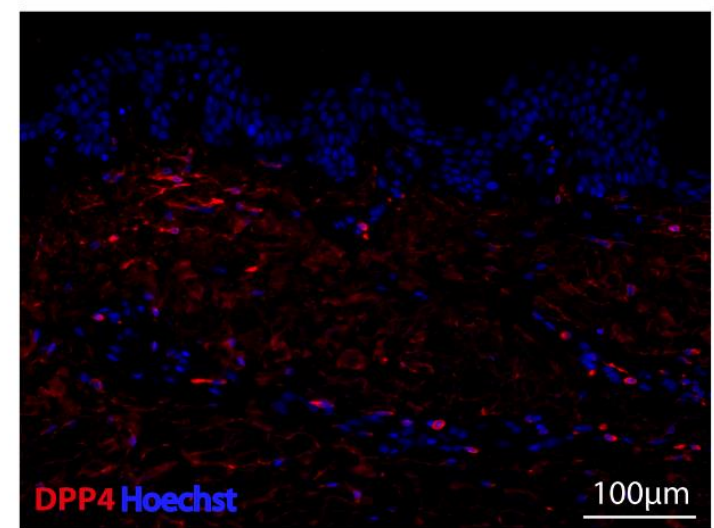

(D)

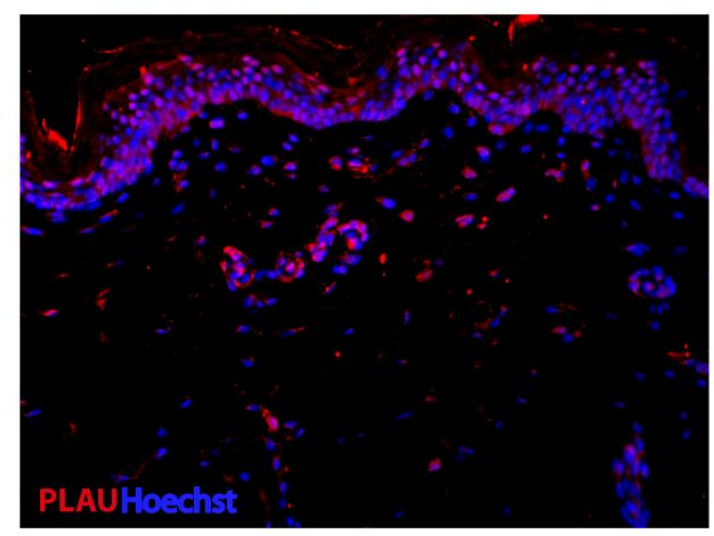

Scar

Figure 7
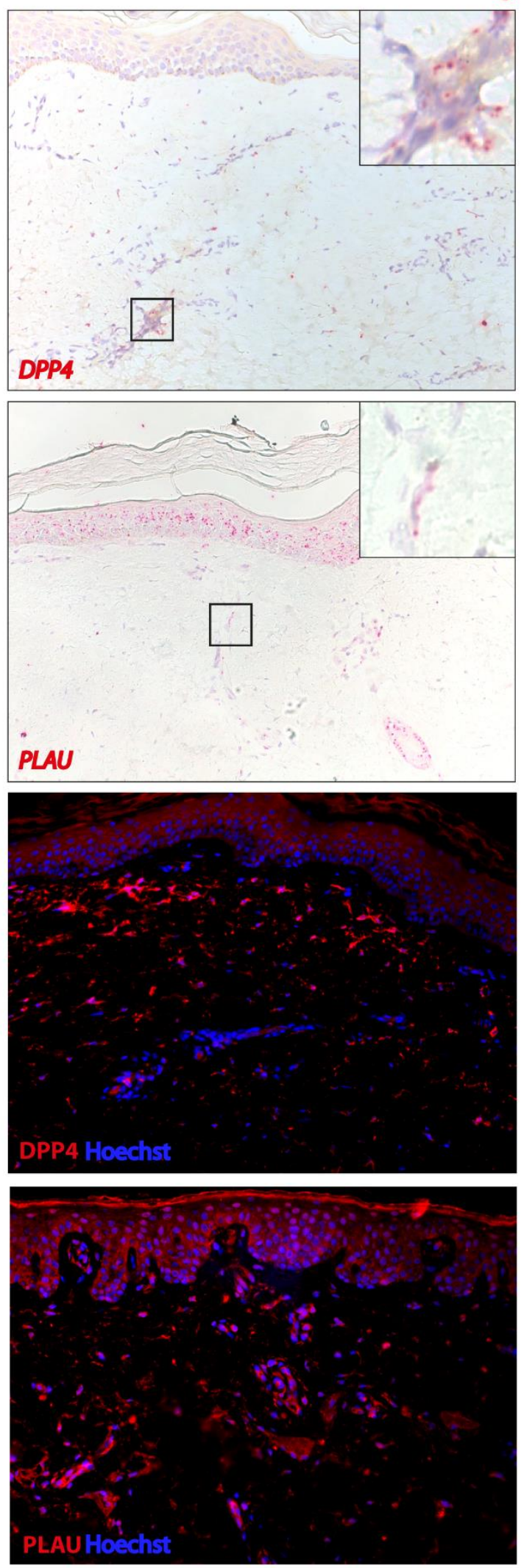

745 Figure 7: In-situ hybridization and immunofluorescence staining confirm elevated expression 746 of $P L A U$ and DPP4 in human skin and scar

747 A, B) RNAScope in-hybridization of skin and scar tissue with DPP4- and PLAU-probes. Red dots 748 indicate single mRNA molecules. Inserts show high magnification micrographs. C, D)

749 Immunofluorescent staining of DPP4 and PLAU in human skin and scar tissues. 
(A)

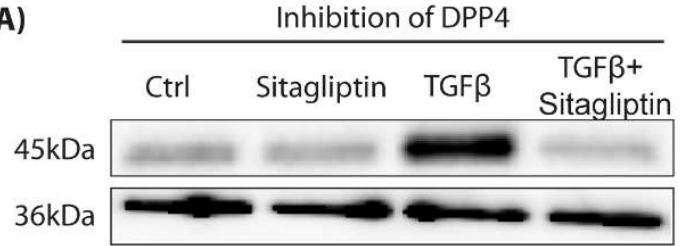

(C)

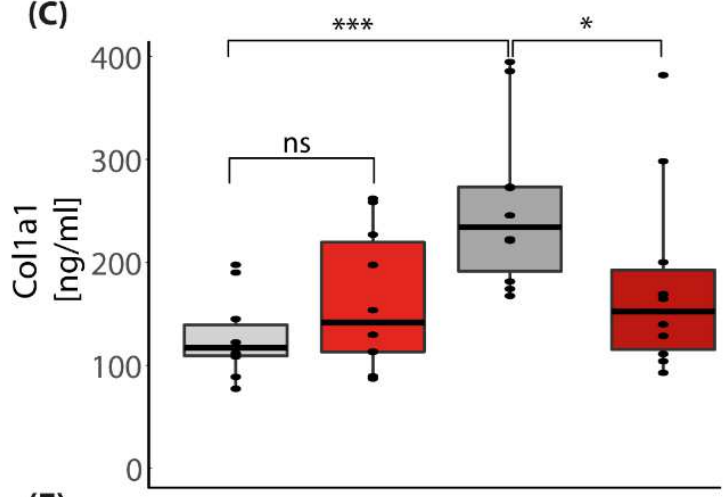

(E)

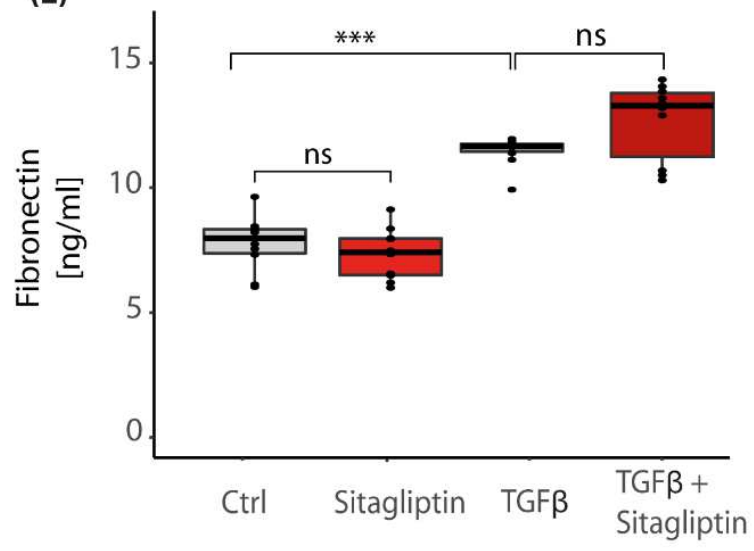

(B)

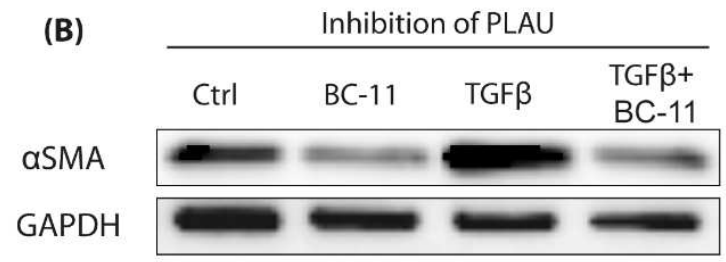

(D)

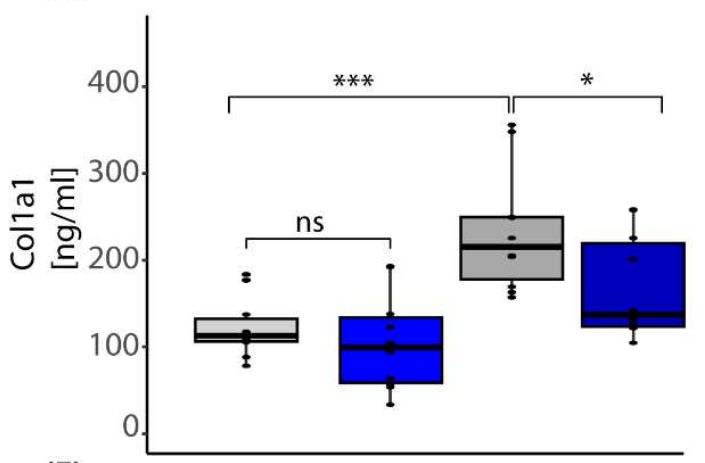

(F)

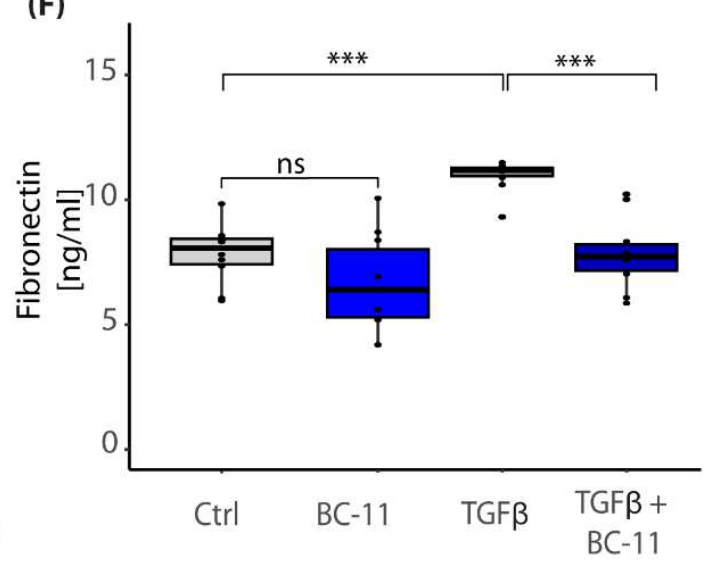

Figure 8: Pharmacological inhibition of myofibroblast-differentiation

A,B) Western blot of primary FBs stimulated with active TGF $\beta 1$ for $24 \mathrm{~h}$ to differentiate FBs into alpha-smooth muscle actin-expressing ( $\alpha$ SMA) myofibroblasts. Myofibroblast differentiation inhibited with A) DPP4-inhibitor Sitagliptin or B) urokinase-inhibitor BC-11. C, D) Collagen I or E, F) fibronectin in supernatants of stimulated primary skin FBs, detected by Enzyme-linked Immunosorbent Assay (ELISA). Whiskers represent range maximum and minimum values with $<1.5$ interquartile range, boxes represent $25^{\text {th }}-75^{\text {th }}$ quartiles, line represents mean. Statistical significance was tested using one-way ANOVA with Tukey post-test. NS $p>0.05,{ }^{*} p<0.05,{ }^{* *} p<0.01,{ }^{* * *} p<0.001$. Experiments were performed in duplicates of five donors each. 


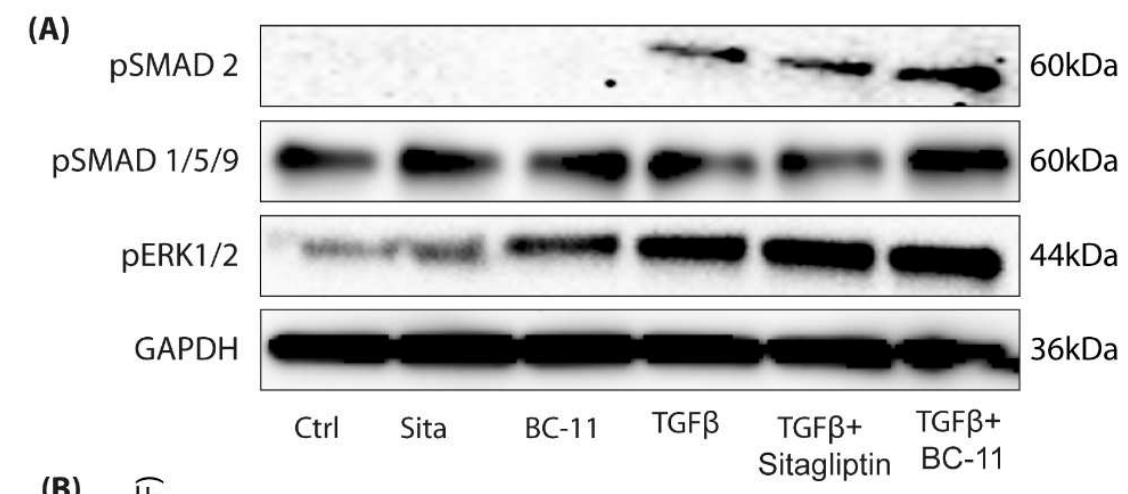

Figure 9

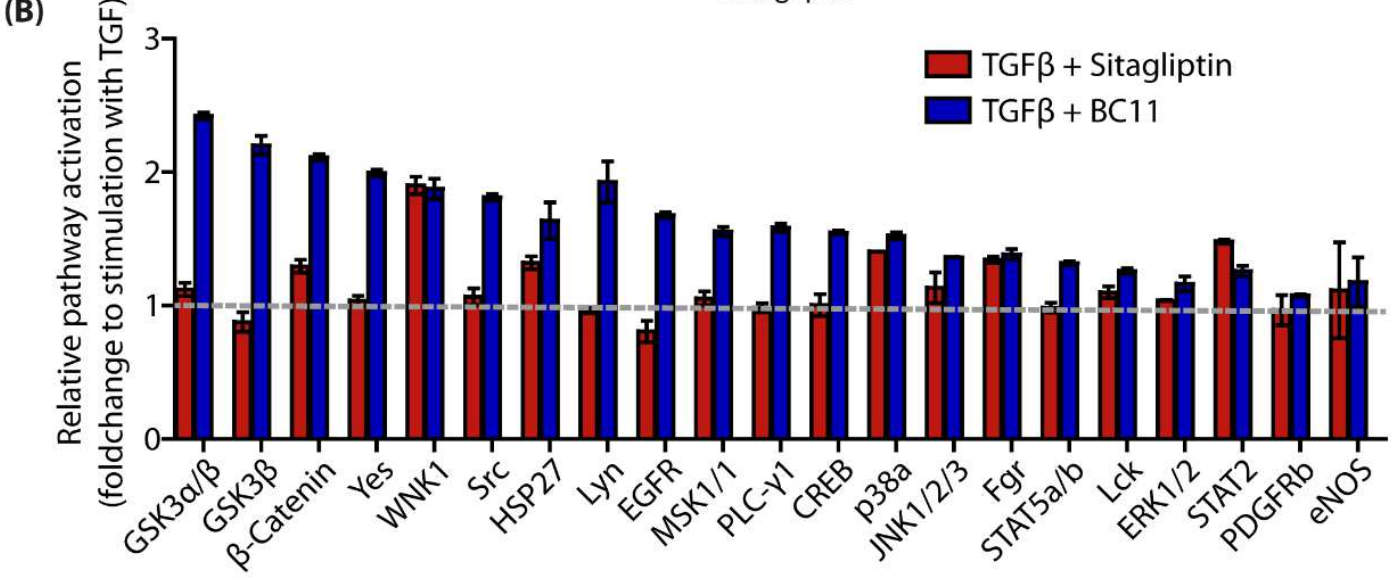

Figure 9: Signaling pathway activation after TGF $\beta$-stimulation and DPP4/PLAU inhibition

E) Western blot of primary FB lysate stimulated with active TGF $\beta 1$ for $1 \mathrm{~h}$ and analysis of canonical TGF $\beta 1$ signaling pathways. B) Analysis of non-canonical TGF $\beta 1$ signaling pathways and kinase pathways detected by proteome profiler from lysates of primary FBs after $1 \mathrm{~h}$ stimulation with TGF $\beta 1$ alone, TGF $\beta 1$ with sitagliptin, and TGF $\beta 1$ with BC-11. Bars represent fold change compared to stimulation with TGF $\beta 1$ only, marked by dotted line. 
(A)

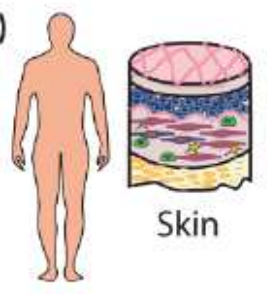

(B)

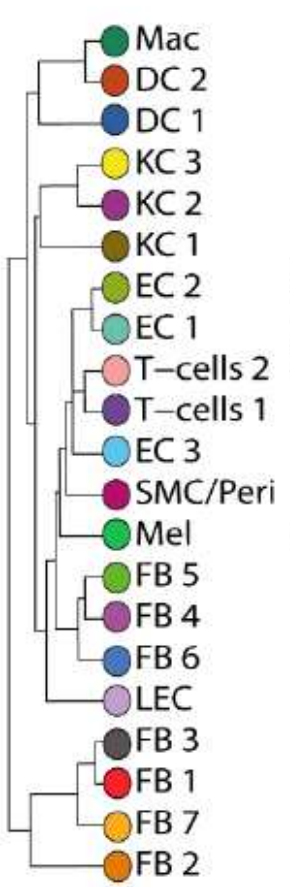

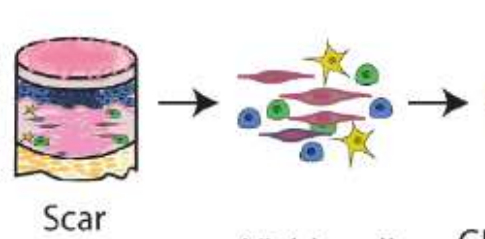

Viable cell suspension

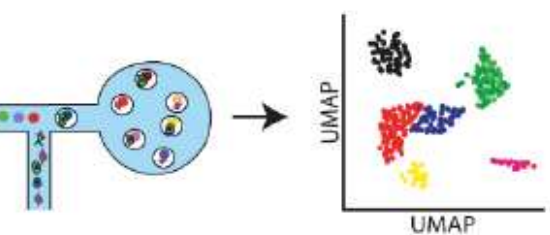

GEM-generation, cDNA-synthesis, sequencing
Gene-Barcode Matrix, Clustering, DEG, Downstream-Analysis
Skin

(C)

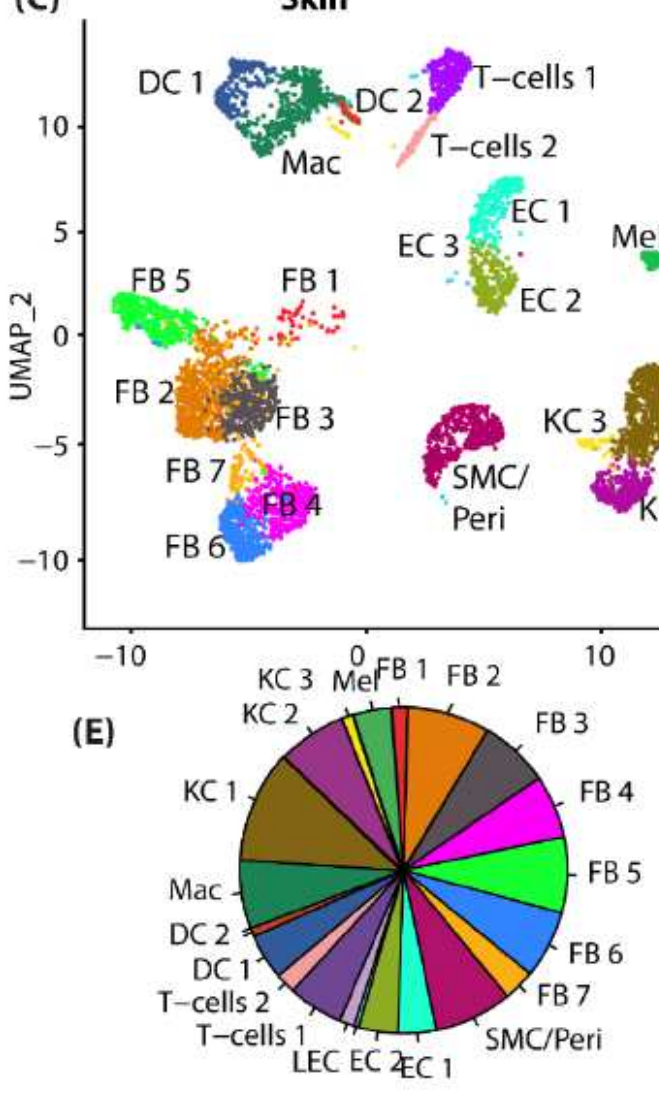

(D)

Scar
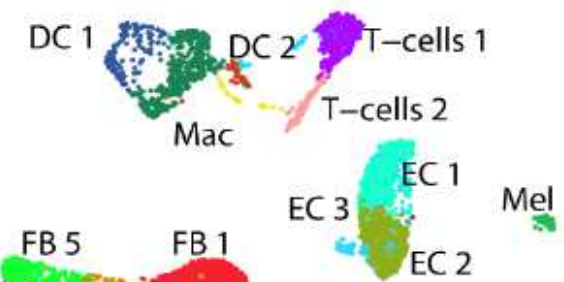

KC 1

FB
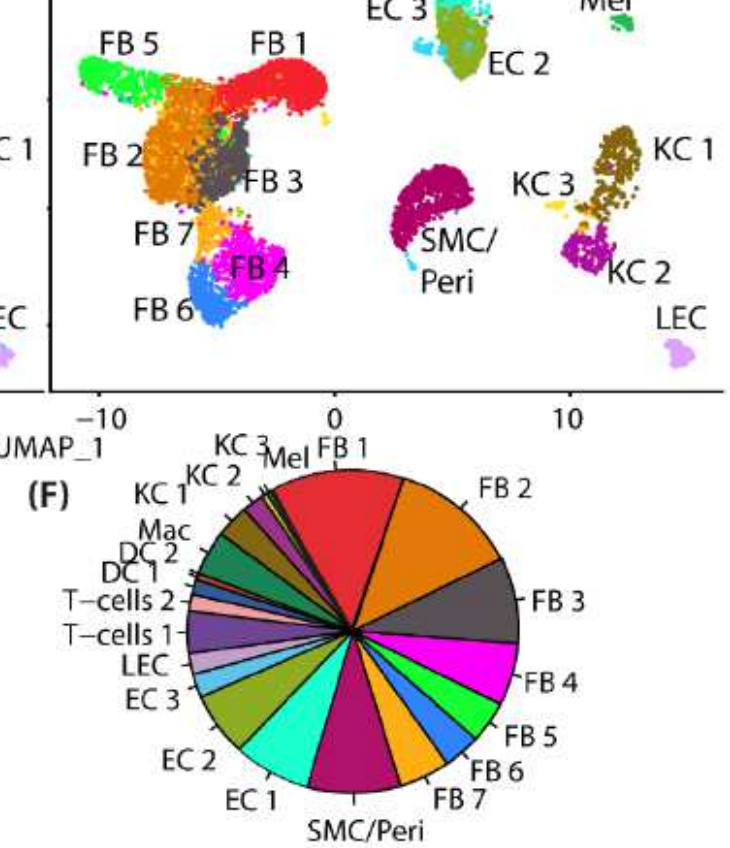

(F)

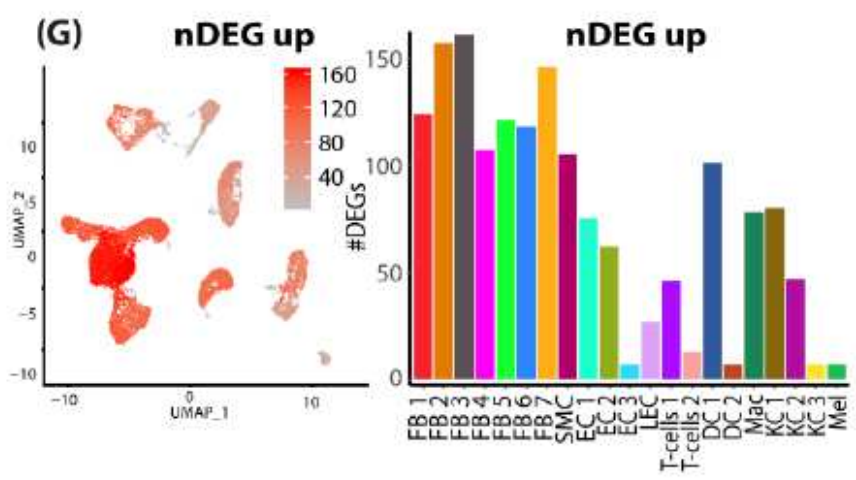

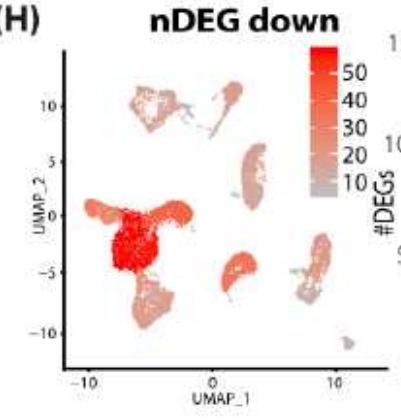

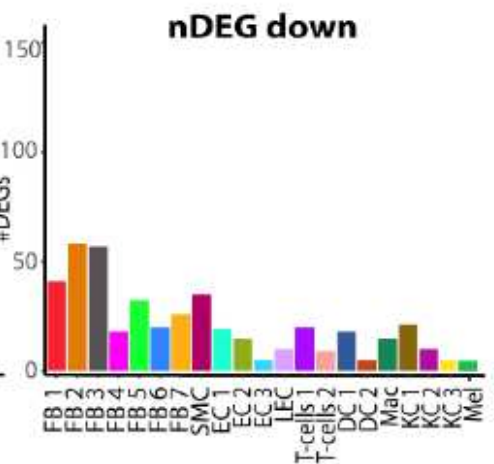

Figure 1

Characterization of human skin and scar samples with scRNAseq identifies specific cell clusters and a distinct fibrotic gene signature. A) Illustration of workflow of scRNAseq in human skin and scar samples. B) Phylogenetic clustertree calculated unsupervised based on UMAP-clustering. C, D) UMAP-plots of 
human skin and scar samples, split by tissue, after integration of all samples, identifying seven fibroblast clusters (FB1-7), smooth muscle cells and pericytes (SMC/Peri), endothelial cells (EC1+2), lymphatic endothelial cells (LEC), T-cells, macrophages (Mac), dendritic cells (DC1+2), three keratinocyte clusters (KC1-3), and melanocytes (Mel). E, F) Pie charts showing ratios of cell clusters in skin and scars. Feature plots and bar graphs of number of differentially expressed genes (nDEG) per cluster of $G$ ) up-and $H$ ) downregulated genes. DEGs were calculated per cluster comparing scar versus skin using Wilcoxon rank sum test, including genes with average logarithmic fold change (avglogFC) of $>0.1$ or $<-0.1$ and Bonferroni-adjusted $p$-value $<0.05$. Feature plots show projection of nDEG onto the UMAP-plot, color intensity represents $\mathrm{nDEG}$. Bar graphs show absolute numbers of nDEG per cluster, $y$-axis represents nDEG. UMAP, uniform manifold approximation and projection. 
(A)

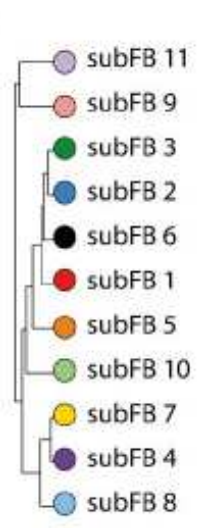

(B) Skin

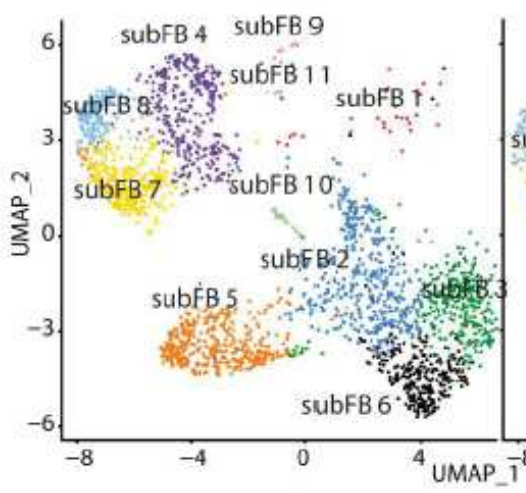

(C) Scar

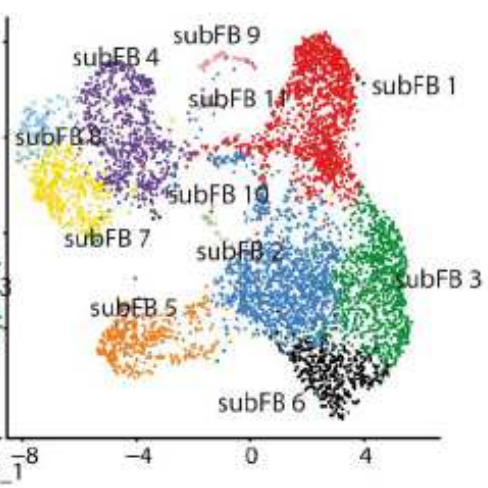

(D)
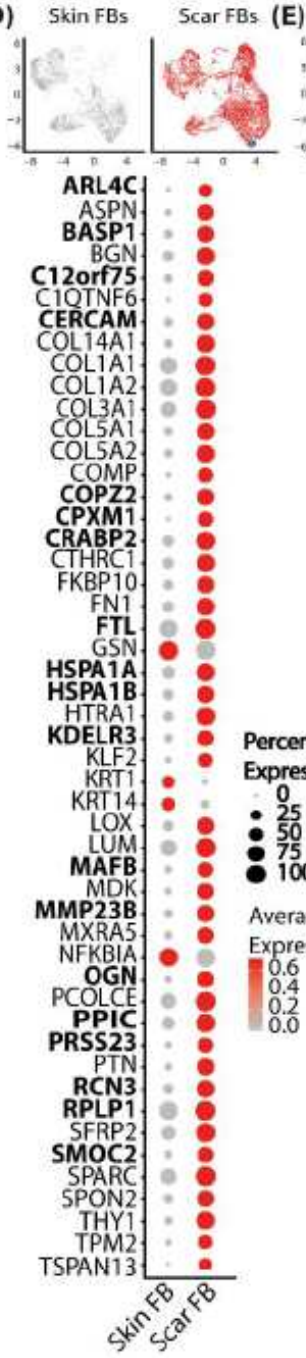
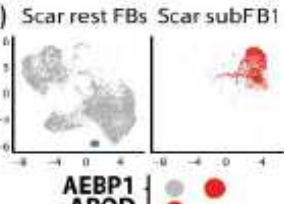

AEBP1 ARLAC ASPN CIOTNF CIQTNFE

CFD

COL12A COL14A1 COL1A COLIA2 COLSA 1 COL5A COMP COPZ2 CTHRC1. CXCL12 CXCL14 $F 10$
FABP5 FGL2 Expressed 0
$: 25$
850
75 750 Average Expression 0.6 0.4
0.2
0.0 FN1
FNDC1 HTRAI LUM

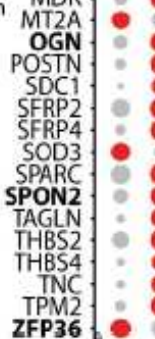

(F)
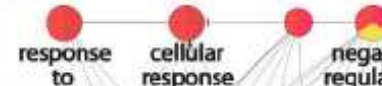
transforming to growth transforming

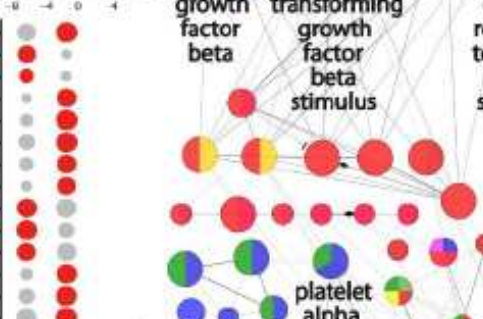
negative extracellular extracellular regulation matrix matrix insulin-like granule o growth binding protein protein
complex
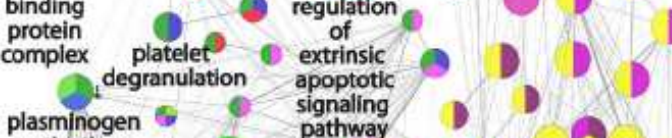
plasminogen activation

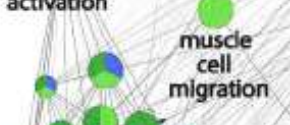
pathwa of organization response to growth factor
stimulus fibrillar positive collar regulation trimer regulation extrinsic $\bigcirc \bigcirc$ glycosaminogly apoptotic
signaling pathway

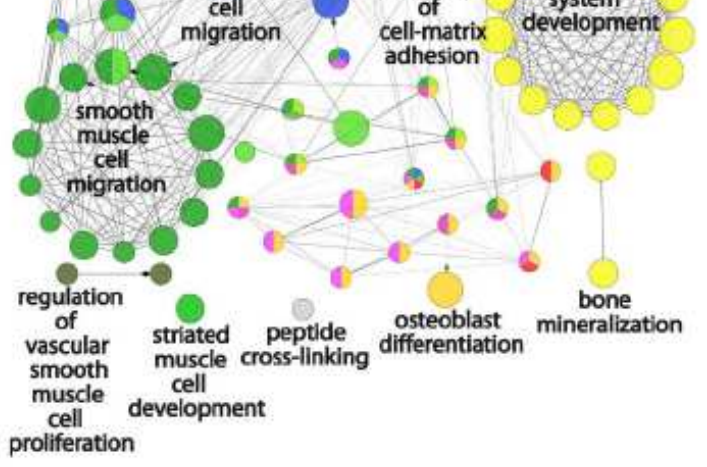

ZFP36 $88^{3}$ कर $5^{0} y^{0}$

\section{Figure 2}




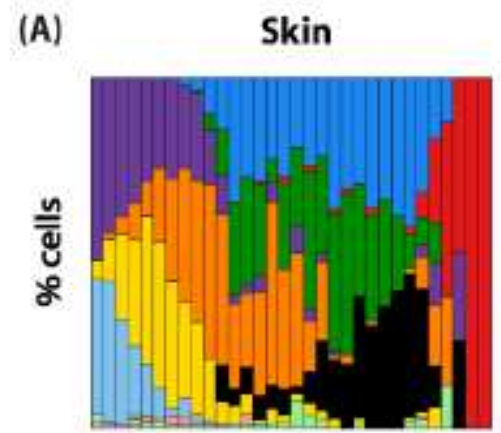

Pseudotime

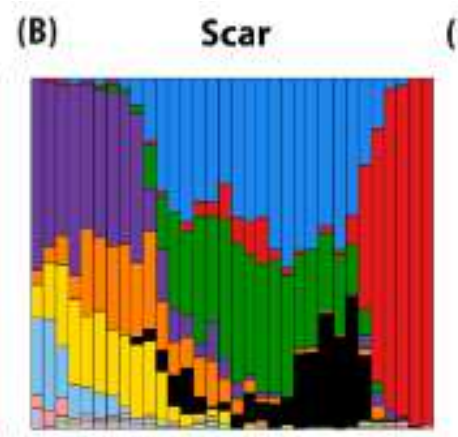

Pseudotime

(C)
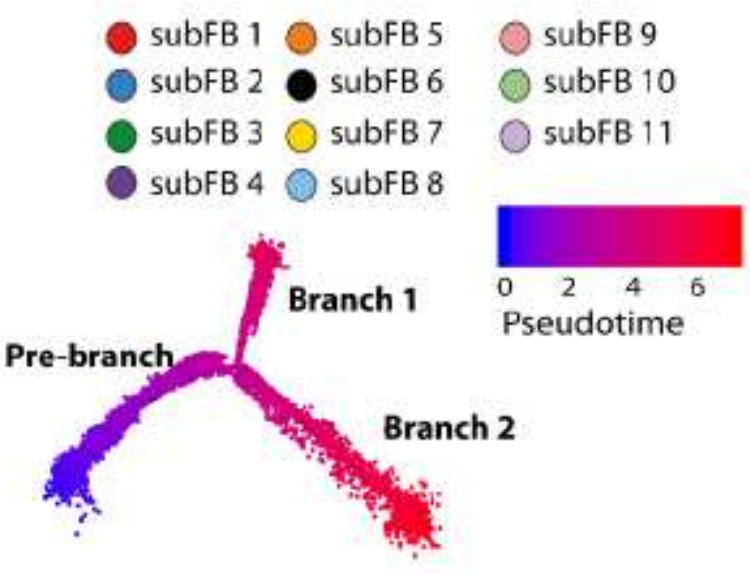

(D)

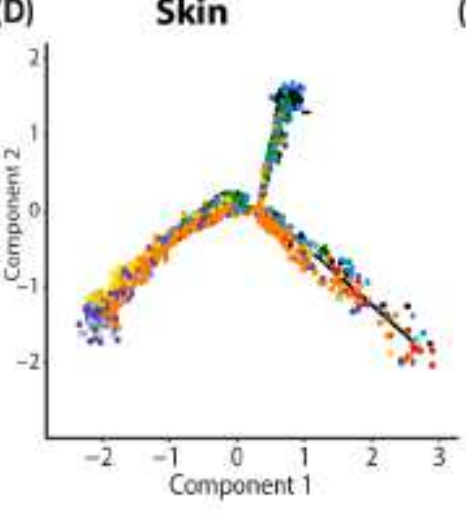

(E)
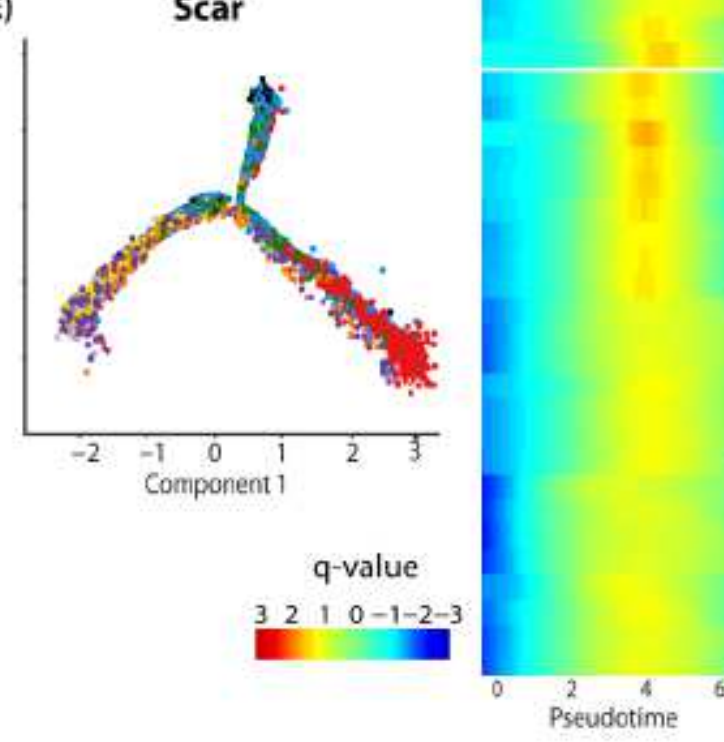

(G) I SPAACL1.1
CCL19 CI CXCLI CXCL1 $\mathrm{CXCL}_{3}$ CXCL2 R.G516 PIGDS SOKS3 GGT5 CrGB CCL 2 GEM APOE
SFRP4 SFRP4 COLIA COLIA1 CLEC3B IGFBPE ELN CIOTNF3 CERCAM SH3BGRL 3 FBN1 PRG4 $\mathrm{CD} 34$ TNXB CDSS MFAP5 CILP HASI SPARC ECM1 SEMA3C SEMA3E SLC29A1 MATN4 CAPS ISPANB CFO CD70 PCSKIN FAM1808 PIGT COL12A WISP2 SLPI PCOLCE2 LOX MMP2 P116 CTHAC1 FBLN2 TIMP2 QPCT DCN PDGFRL DPP4 FBLN1 SEARA5 MFGE\& MiFGE8

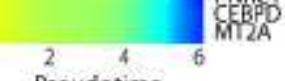

Pseudotime

\section{Figure 3}

Pseudotime analysis of human scar FBs identifies cell fates and time-regulated genes A, B) Ordering skin and scar FBs along a pseudotime axis. X-axis, pseudotime. y axis, \% of cells in respective pseudotime-bin. Cell trajectory with pre-branch and branches is shown. Color code represents pseudotime progression. C, D) Cell trajectories were calculated based on pseudotime values, split by tissue. E, F) Heatmaps of pseudotime-dependent gene expression in skin and scar. Colors represent q-value, the expression of the respective gene in relation to pseudotime. 
(A)

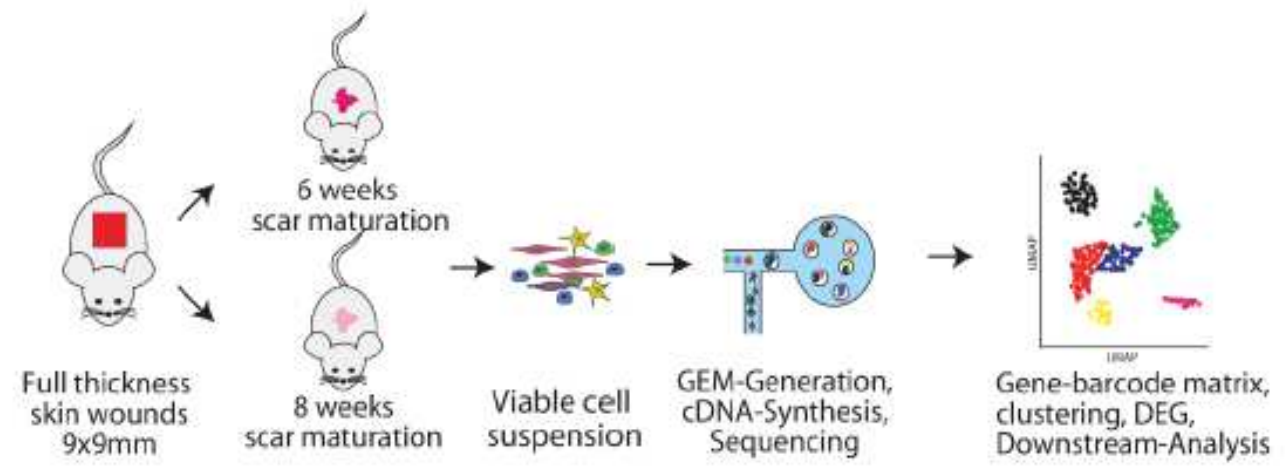

(B)

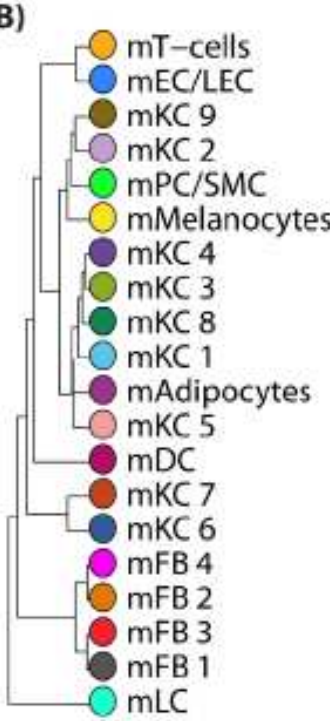

(C) 6 weeks

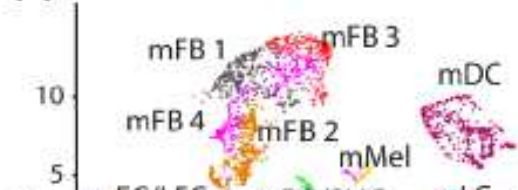

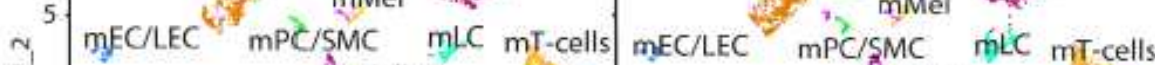

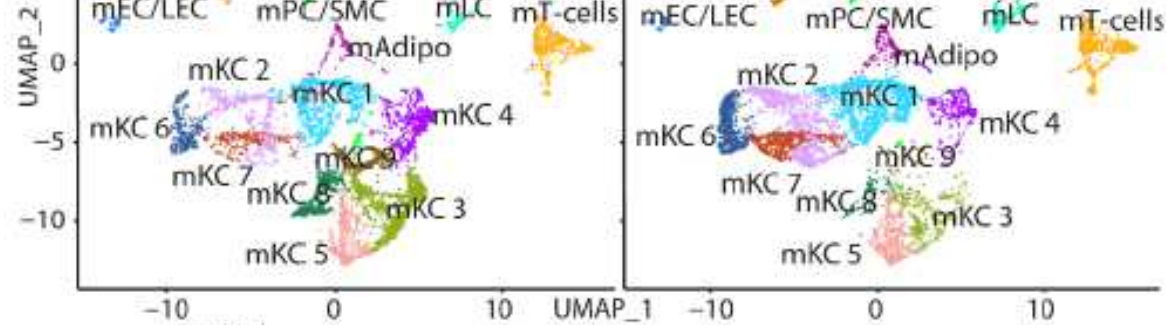

(D)

8 weeks
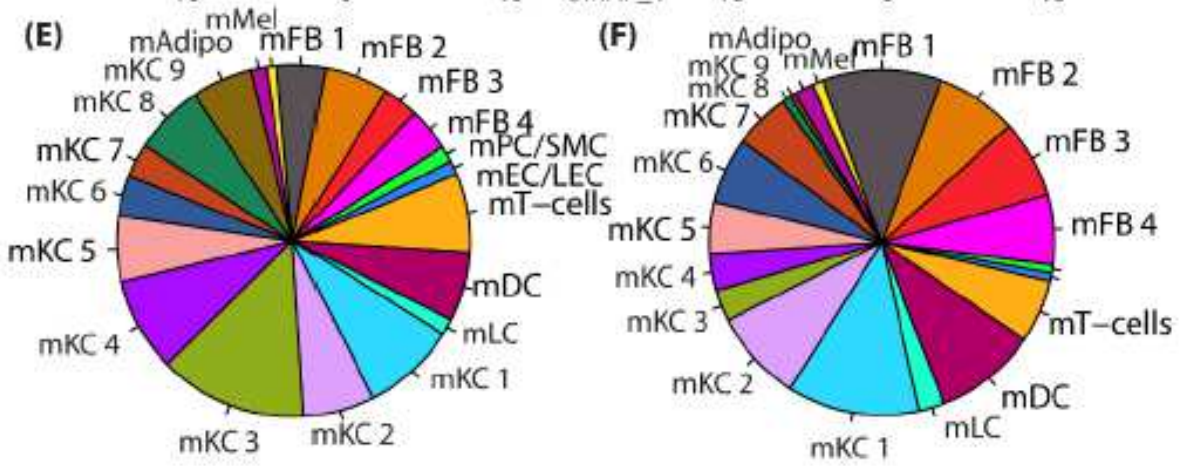

(G)
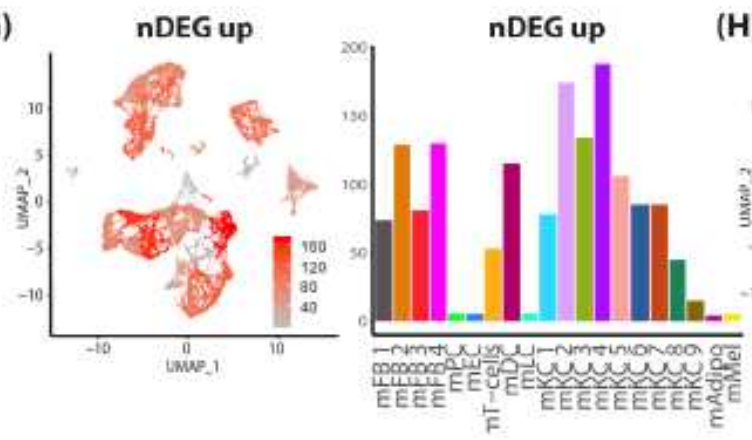

(H)
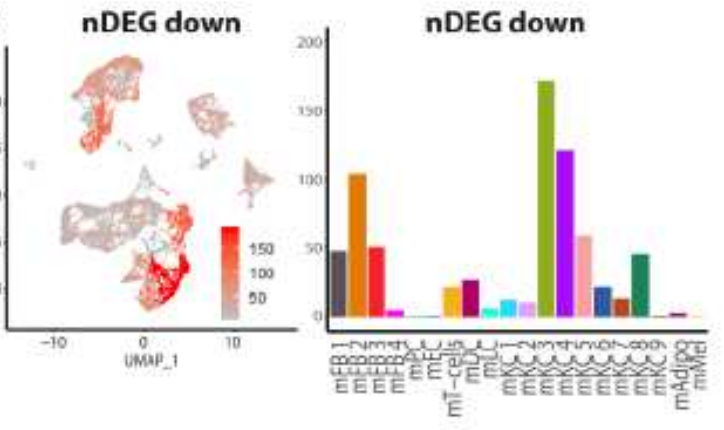

\section{Figure 4}

Two-timepoint mouse scar model identifies genes regulated in scar maturation A) Illustration of workflow of mouse scar model and two-timepoint scRNAseq. Two mice were analyzed per timepoint. B) Phylogenetic clustertree calculated unsupervised based on unsupervised UMAP-clustering. C, D) UMAPplots of mouse scar tissue, split by timepoint, after integration of all samples, identifying four fibroblast clusters (mFB1-4), smooth muscle cells and pericytes (mPC/SMC), endothelial cells and lymphatic 
endothelial cells ( $\mathrm{mEC} / \mathrm{LEC}), \mathrm{T}$-cells, dendritic cells $(\mathrm{mDC})$, Langerhans cells $(\mathrm{mLC})$, nine keratinocyte clusters (KC1-9), adipocytes (mAdipo) and melanocytes (Mel). E, F) Pie charts show relative numbers of cells in clusters, split by timepoint. Feature plots and bar graphs of number of differentially expressed genes ( $\mathrm{nDEG}$ ) per cluster of $\mathrm{G}$ ) up- and $\mathrm{H}$ ) downregulated genes per cluster. DEGs were calculated per cluster comparing 8 weeks vs 6 weeks old scars using Wilcoxon rank sum test, including genes with average logarithmic fold change (avg_logFC) of $>0.1$ or $<-0.1$ and Bonferroni-adjusted $p$-value $<0.05$. Feature plots show projection of nDEG onto the UMAP-plot, color intensity represents nDEG. Bar graphs show absolute number of nDEG per cluster, y-axis represents nDEG. UMAP, uniform manifold approximation and projection. 
(A)

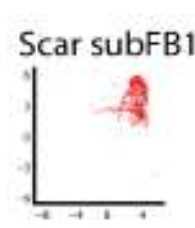

Scar rest FB
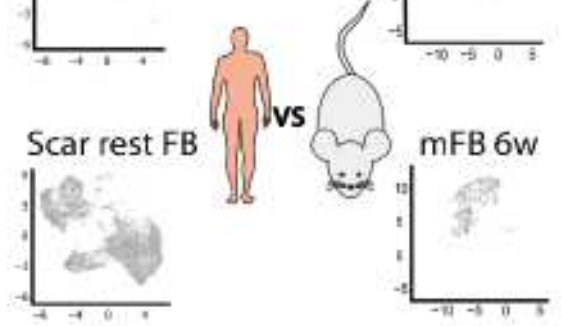

(B)

subFB1 vs rest scarFB
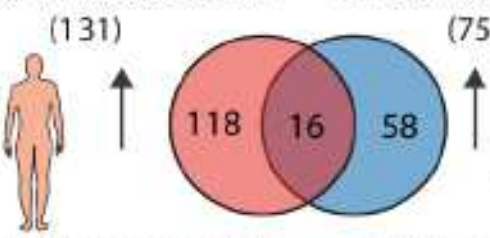

(75)

subFB1 vs rest scarFB

(179)

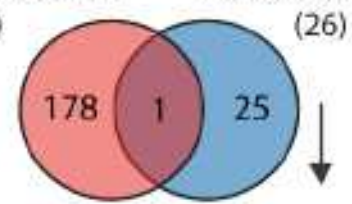

(C)

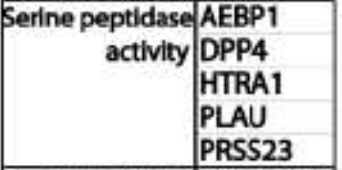

Extracellular COL14A1 matrix FNDC1 components LUM TGFbeta OGN signaling TGFBI

Insulin-like IGFBP3 growth-factor IGFBP6 various FIBIN HSPA1A RARRES2 SFRP4

(D)

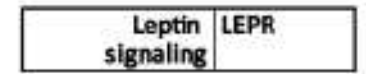

Scar

(E)

(F)

Skin
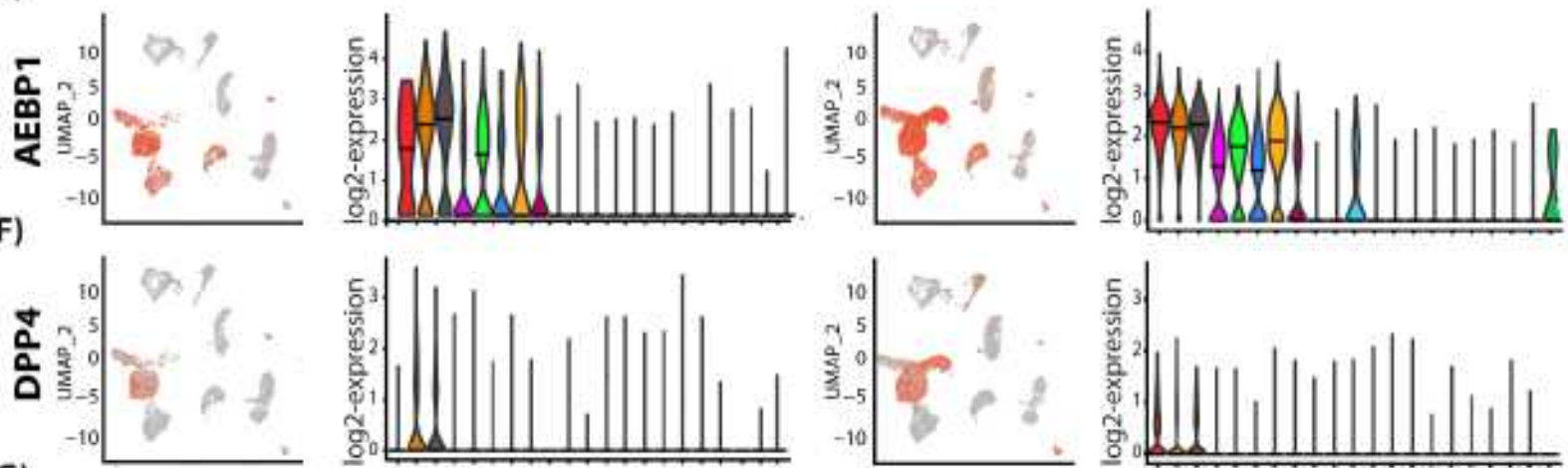

(G)
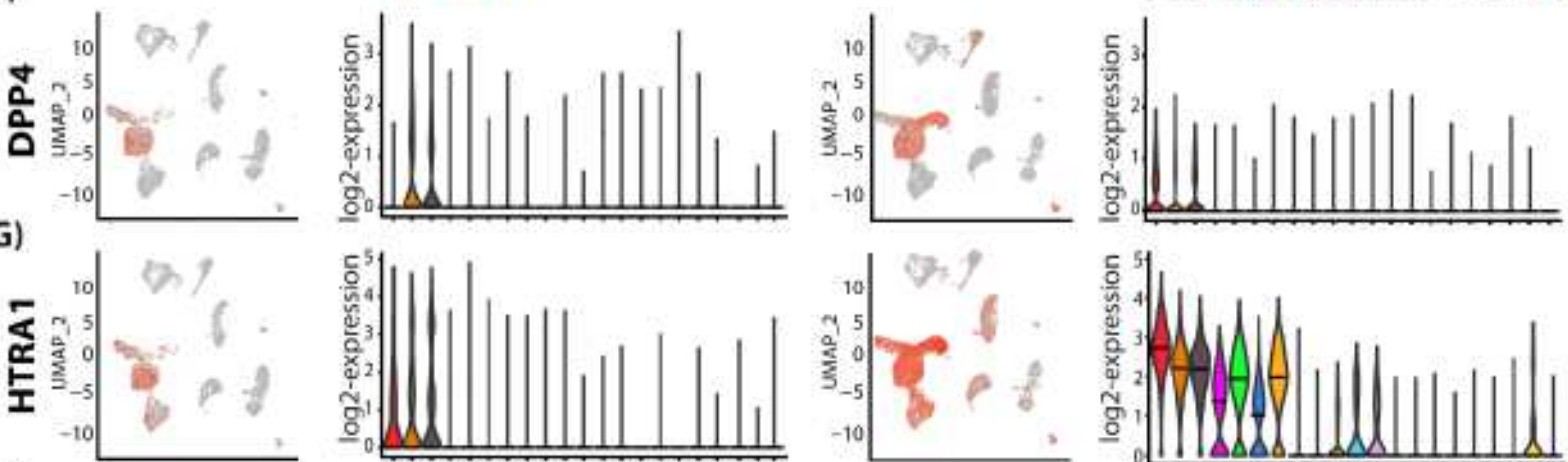

(H)
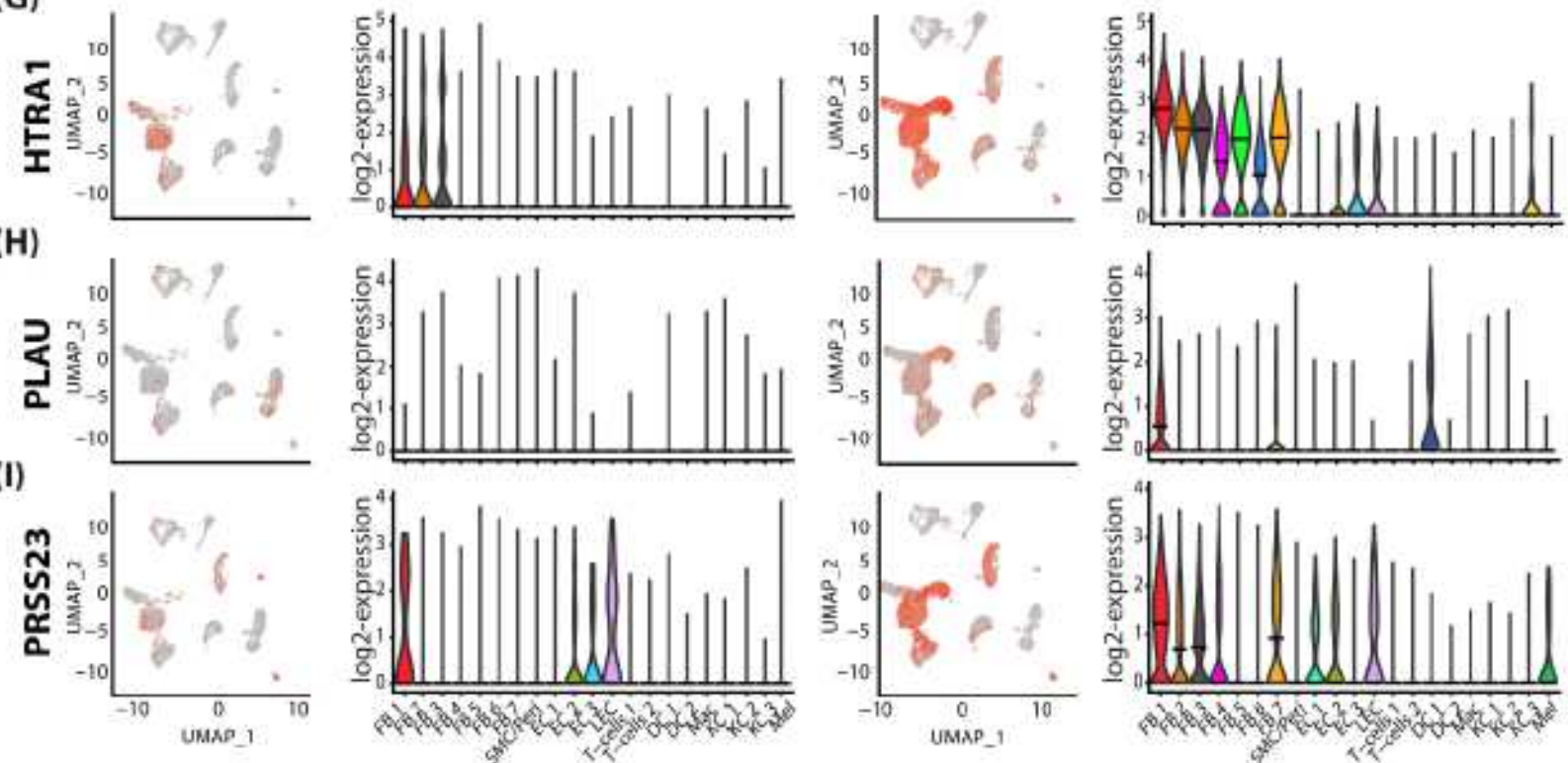

\section{Figure 5}

Comparing human scar gene expression and mouse scar maturation identifies mutual drivers of skin fibrosis A) Illustration of computational basis for comparison human and mouse. Human cluster subFB1 vs rest scar FBs significantly (adj. p-value $<0,05$ ) regulated genes were compared with mouse scar FBs 8 weeks vs 6 weeks significantly regulated genes. B) Venn diagrams of human and mouse up- (upper panel) and down- (lower panel) regulated genes. C) Table of mouse and human mutually up and D) 
downregulated genes. E-I) Feature plots and violin plots of serine proteases in human skin and scar. AEBP1 (adipocyte enhancer binding protein 1), DPP4 (dipeptidyl-peptidase 4), HTRA1 (High-Temperature Requirement A Serine Peptidase 1), PLAU (urokinase), PRSS23 (Serine protease 23). In violin plots, dots represent individual cells, $y \llbracket$ axis represents log2 fold change of the normalized genes and $\log \rrbracket$ transformed single『cell expression. Vertical lines in violin plots represent maximum expression, shape of each violin represents all results, and width of each violin represents frequency of cells at the respective expression level. In feature plots, normalized log expression of the respective gene is mapped onto the UMAP\plot. Color intensity indicates level of gene expressions. UMAP, uniform manifold approximation and projection. 
(A)

Skin

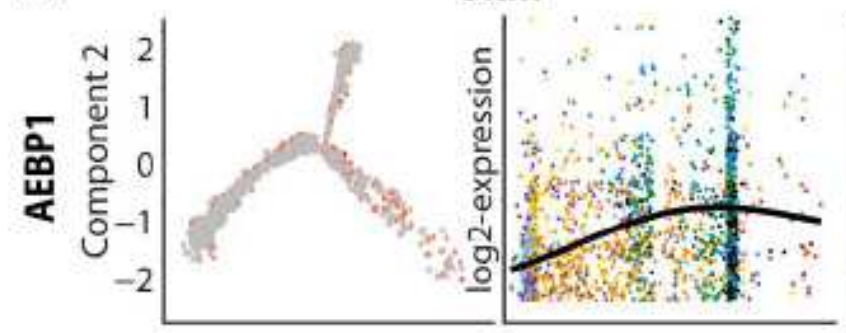

(B) 글

(C)
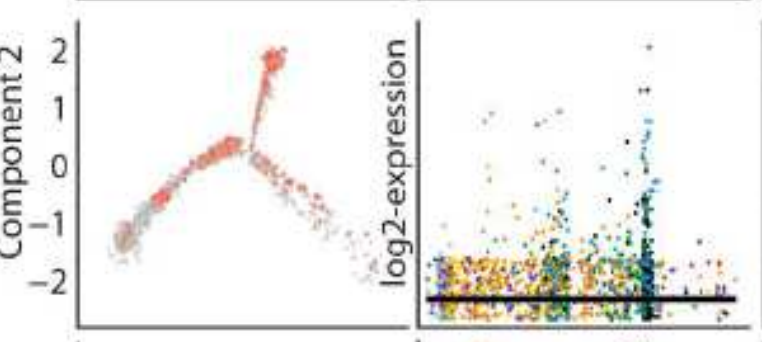

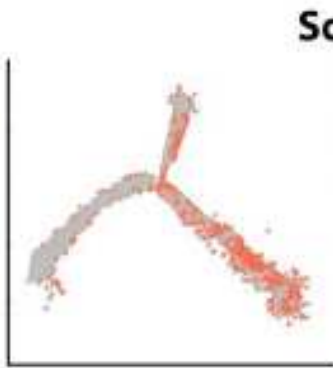

Scar
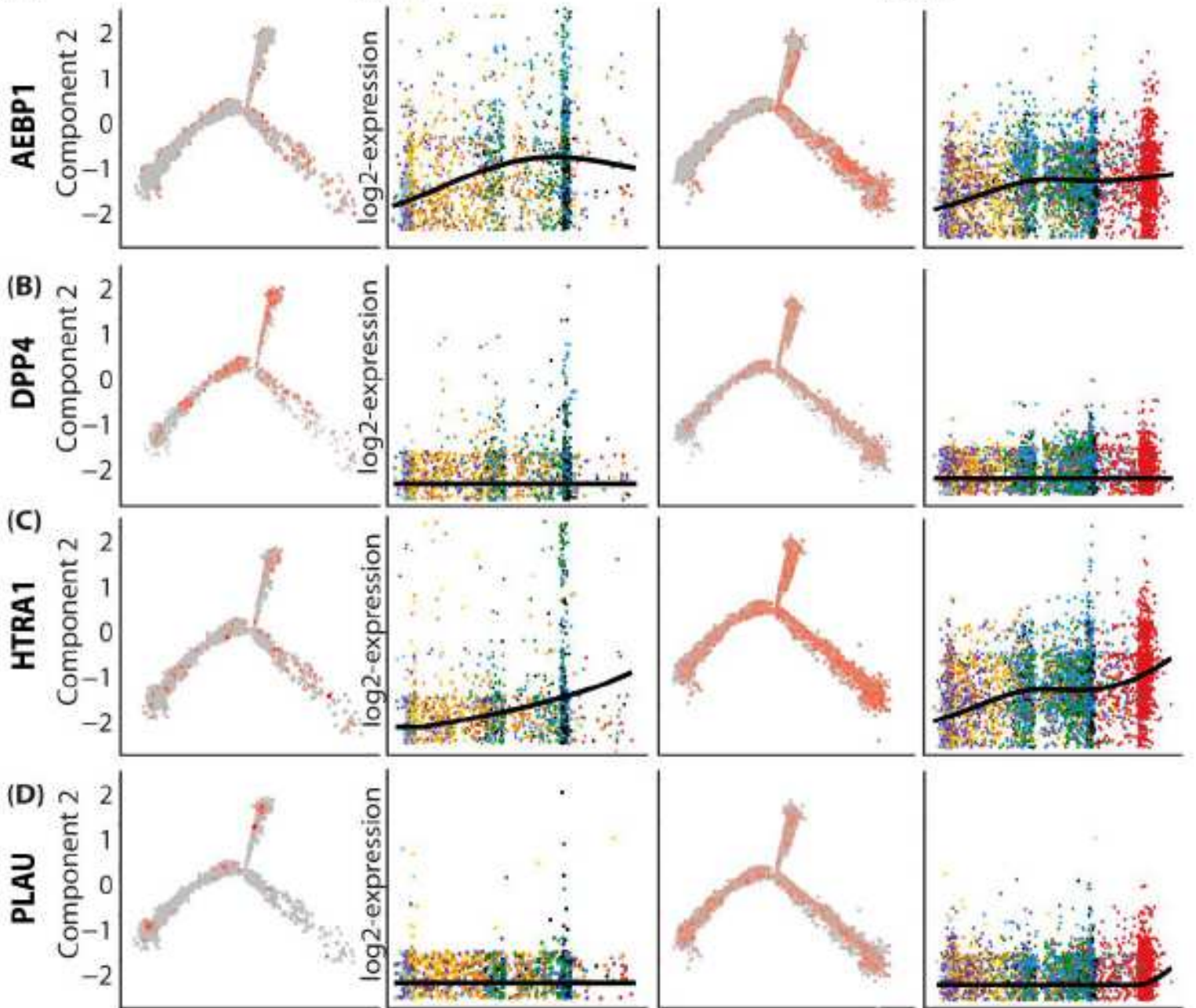

(E)
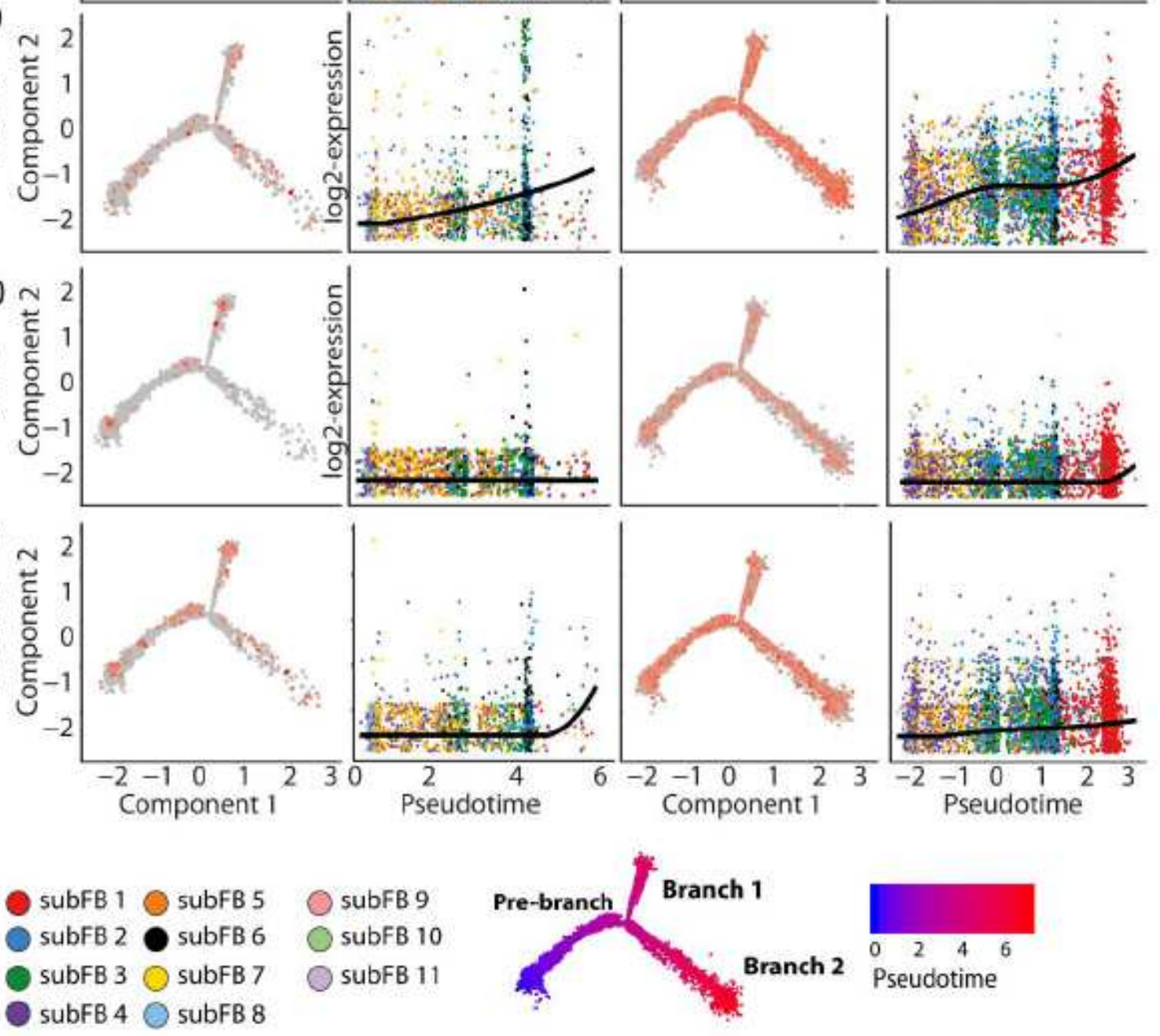

subFB 4 subFB 8

\section{Figure 6}

Pseudotime analysis corroborates the putative role of serine proteases as drivers of scar maturation A-E) Trajectory plots and pseudotime plots of Serine proteases in skin and scar FBs. AEBP1 (adipocyte enhancer binding protein 1), DPP4 (dipeptidyl-peptidase 4), HTRA1 (High-Temperature Requirement A Serine Peptidase 1), PLAU (urokinase), PRSS23 (Serine protease 23). In trajectory plots, normalized log expressions are plotted on the trajectories, split by skin and scar. In pseudotime plots, normalized log 
expressions are plotted against the pseudotime axis, and a spline curve represents expression dynamics over pseudotime. Y-axis, normalized log expression of respective gene, x-axis, pseudotime.

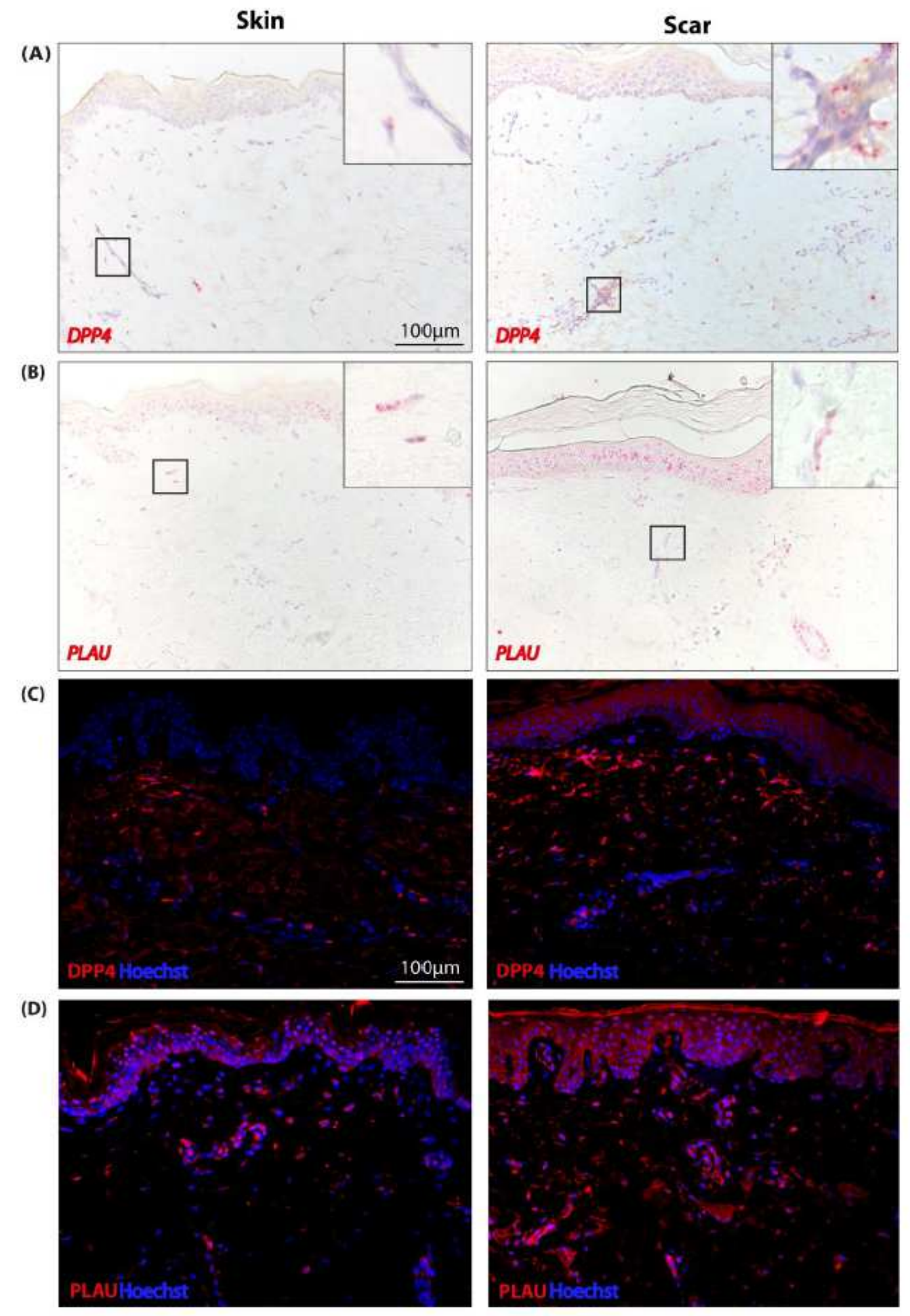

Figure 7

In-situ hybridization and immunofluorescence staining confirm elevated expression of PLAU and DPP4 in human skin and scar A, B) RNAScope in-hybridization of skin and scar tissue with DPP4- and PLAU- 
probes. Red dots indicate single mRNA molecules. Inserts show high magnification micrographs. C, D) Immunofluorescent staining of DPP4 and PLAU in human skin and scar tissues.

(A)
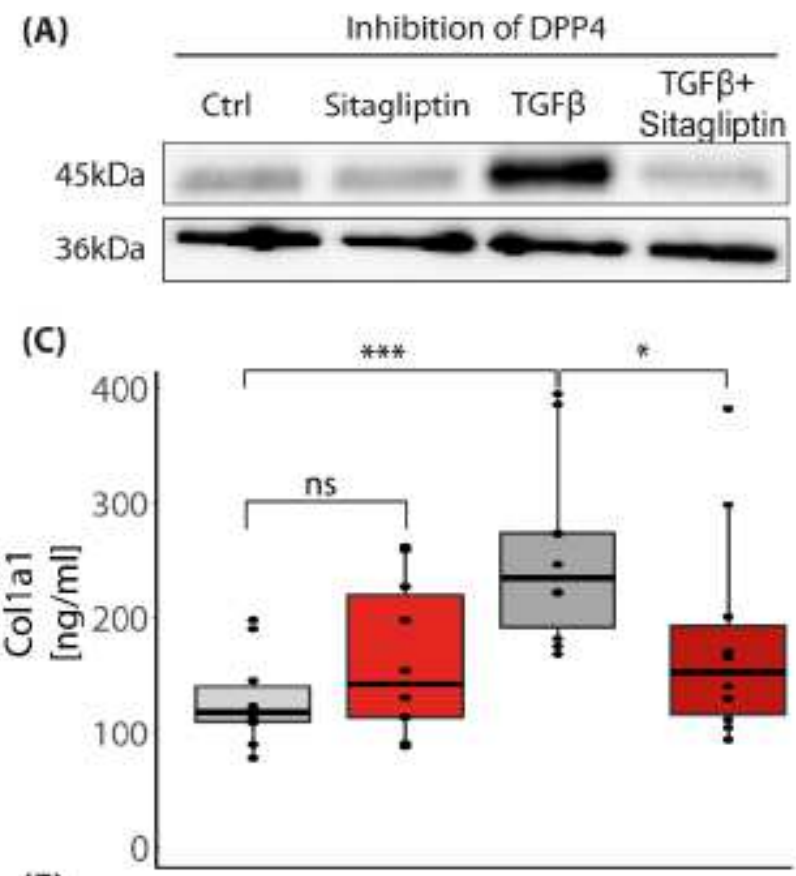

(E)

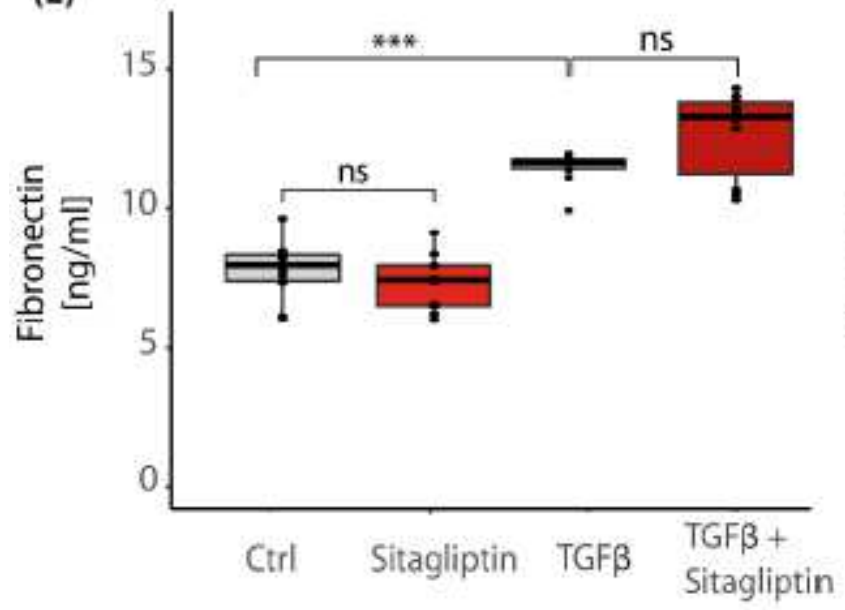

(B)

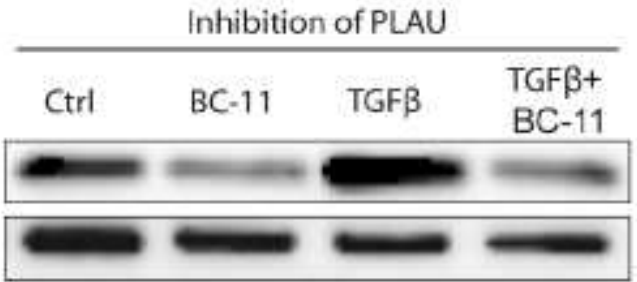

(D)

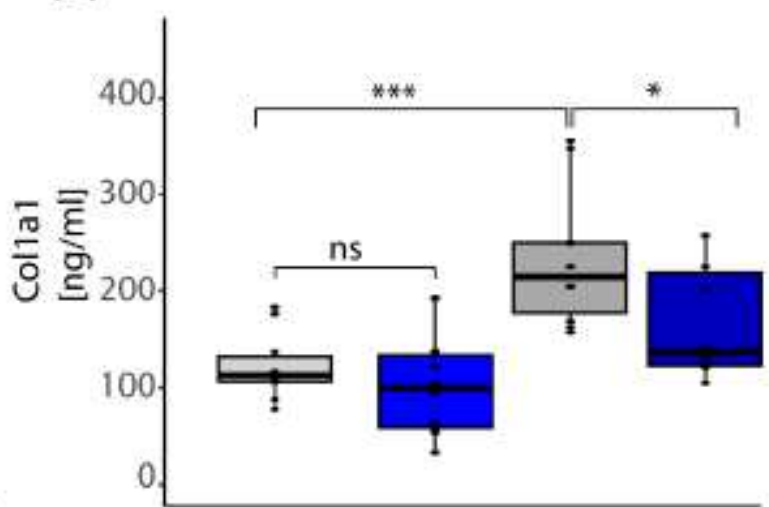

(F)

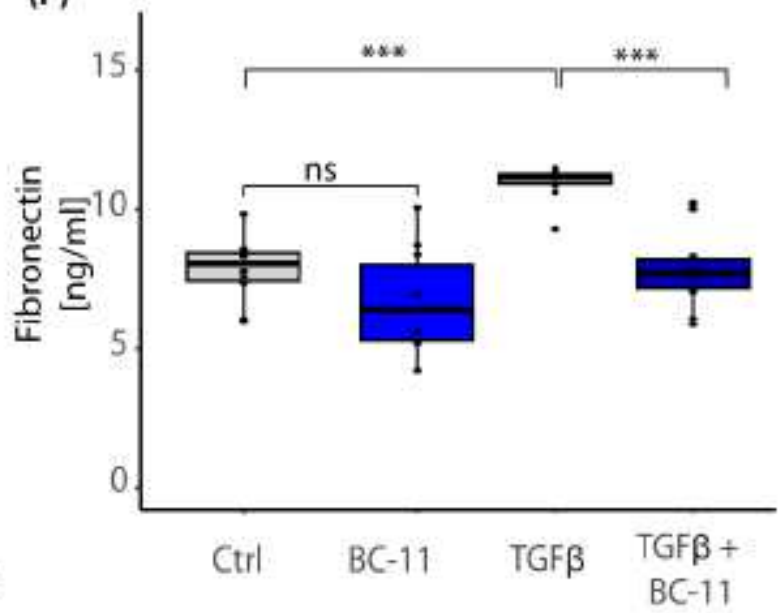

Figure 8

Pharmacological inhibition of DPP4 or urokinase prevents TGF $\beta$-induced myofibroblast-differentiation $A, B)$ Western blot of primary FBs stimulated with active TGF $\beta 1$ for $24 \mathrm{~h}$ to differentiate FBs into alphasmooth muscle actin-expressing (aSMA) myofibroblasts. Myofibroblast differentiation inhibited with A) DPP4-inhibitor Sitagliptin or B) urokinase-inhibitor BC-11. C, D) Collagen I or E, F) fibronectin in supernatants of stimulated primary skin FBs, detected by Enzyme-linked Immunosorbent Assay (ELISA). Whiskers represent range maximum and minimum values with $<1.5$ interquartile range, boxes represent 25th -75 th quartiles, line represents mean. Statistical significance was tested using one-way ANOVA with Tukey post-test. NS $p>0.05,{ }^{*} p<0.05,{ }^{* \star} p<0.01,{ }^{\star \star \star} p<0.001$. Experiments were performed in duplicates of five donors each. 
(A)

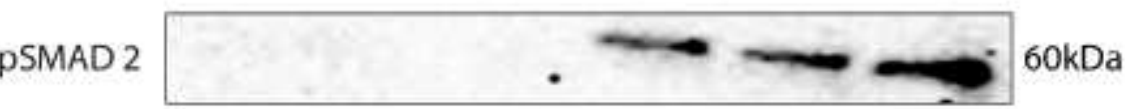

pSMAD $1 / 5 / 9$

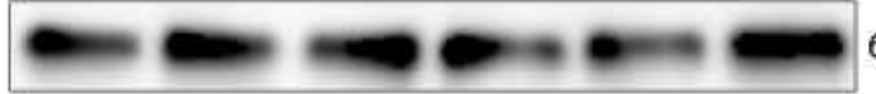

$60 \mathrm{kDa}$

pERK $1 / 2$

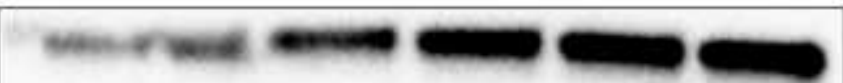

$44 \mathrm{kDa}$

GAPDH

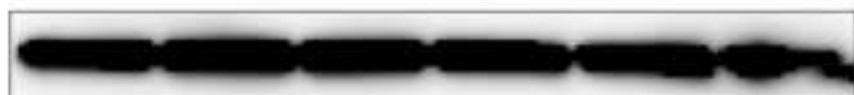

$36 \mathrm{kDa}$

Ctrl Sita BC-11 TGF $\beta \begin{array}{cc}\text { TGF } \beta+ & \text { TGF } \beta+ \\ \text { Sitagliptin } & B C-11\end{array}$

(B)

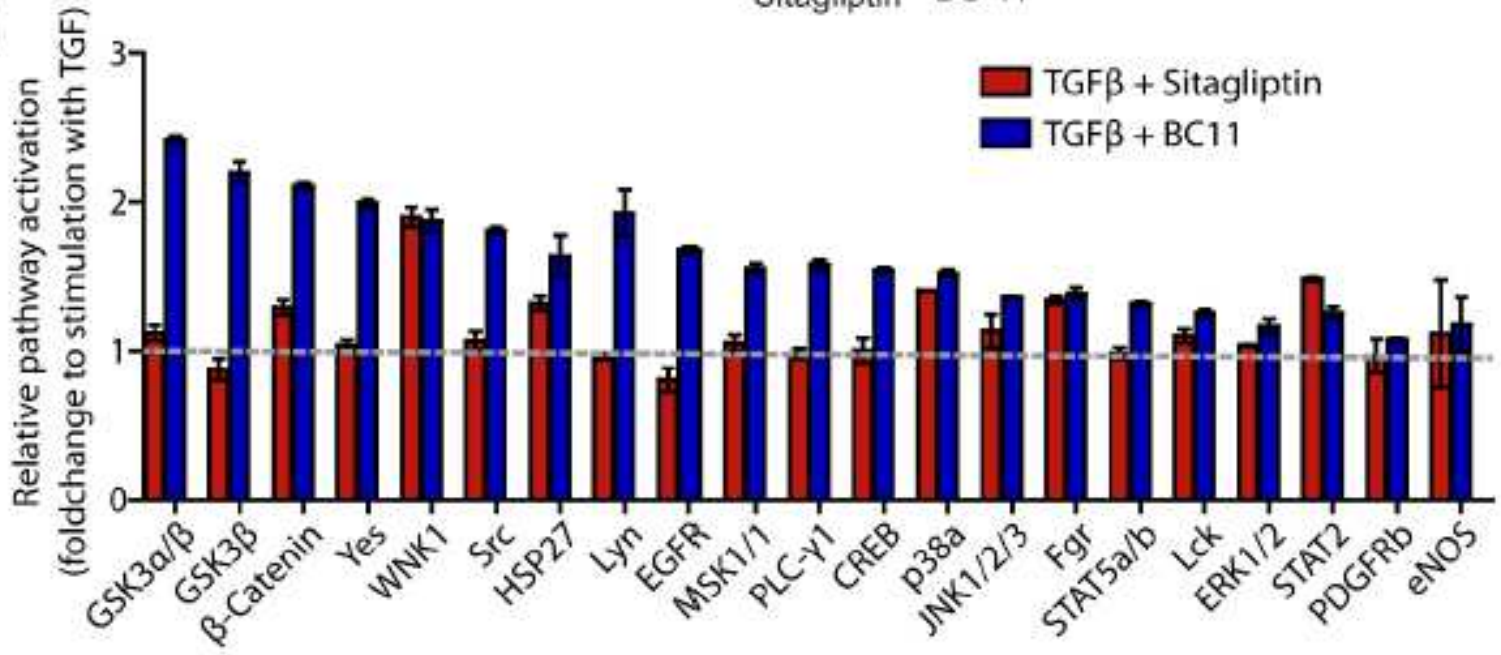

Figure 9

Signaling pathway activation after TGF $\beta$-stimulation and DPP4/PLAU inhibition E) Western blot of primary FB lysate stimulated with active TGF $\beta 1$ for $1 \mathrm{~h}$ and analysis of canonical TGF $\beta 1$ signaling pathways. B) Analysis of non-canonical TGF $\beta 1$ signaling pathways and kinase pathways detected by proteome profiler from lysates of primary FBs after 1h stimulation with TGF $\beta 1$ alone, TGF $\beta 1$ with sitagliptin, and TGF $\beta 1$ with BC-11. Bars represent fold change compared to stimulation with TGF $\beta 1$ only, marked by dotted line.

\section{Supplementary Files}

This is a list of supplementary files associated with this preprint. Click to download.

- 20200805ScarvsskinSupplementarymaterialnatcommsubmission.pdf

- Accesstodata.docx 